\title{
PIP5k1 $\beta$ controls bone homeostasis through modulating both osteoclast and osteoblast differentiation
}

Xiaoying Zhao ${ }^{1,2}$, Guoli $\mathrm{Hu}^{1,2}$, Chuandong Wang ${ }^{1}$, Lei Jiang ${ }^{4}$, Jingyu Zhao ${ }^{1,2}$, Jiake $\mathrm{Xu}^{3}$, Xiaoling Zhang ${ }^{1,2 *}$

${ }^{1}$ Department of Orthopedic Surgery, Xinhua Hospital, School of Medicine, Shanghai Jiao Tong University, Shanghai 200092, China

${ }^{2}$ The Key Laboratory of Stem Cell Biology, Shanghai Institutes for Biological Sciences, Chinese Academy of Sciences, Shanghai 200025, China

${ }^{3}$ School of Pathology and Laboratory Medicine, University of Western Australia, Perth, Western Australia, 6009, Australia

${ }^{4}$ Key laboratory of Tibetan medicine research, Northwest Plateau Institute of Biology, Chinese Academy of Sciences, Xining 810001, PR China

*Corresponding author: Department of Orthopedic Surgery, Xin Hua Hospital, School of Medicine, Shanghai Jiao Tong University (SJTUSM), Shanghai 200092, China. Tel: +86 21 54923338; Fax: +86 21 54923338; E-mail: xlzhang@ shsmu.edu.cn 
Abstract: PIP5K1 $\beta$ is crucial to generation of phosphotidylinosotol $(4,5) \mathrm{P}_{2}$. PIP5K1 $\beta$ participates in numerous cellular activities, such as B cell and platelet activation, cell phagocytosis and endocytosis, cell apoptosis, and cytoskeletal organization. In the present work, we aimed to make insight into the function of PIP5K1 $\beta$ in osteoclastogenesis and osteogenesis to provide promising strategies for osteoporosis prevention and treatment. We discovered that PIP5k1 $\beta$ deletion in mice resulted in obvious bone loss and PIP5K1 $\beta$ was highly expressed both during osteoclast and osteoblast differentiation, besides, PIP5K1 $\beta$ deletion enhanced the proliferation and migration of BMMs to promote osteoclast differentiation. PIP5k1 $1 \beta^{-/-}$ osteoclast exhibited normal cytoskeleton architecture but stronger resorption activity. PIP5k1 $\beta$ deficiency also promoted activation of MAPK and Akt signaling, enhanced TRAF6 and c-Fos expression, facilitated the expression and nuclear translocation of NFATC1 and upregulated Grb2 expression, thereby accelerating osteoclast differentiation and function. Finally, PIP5K1 $\beta$ enhanced osteoblast differentiation by upregulating master genes expression through triggering smad1/5/8 signaling. Thereby, PIP5K1 $\beta$ modulate bone homeostasis and remodeling.

Keywords: PIP5k1 $\beta$, osteoclast differentiation, osteoblast differentiation, proliferation, migration, NFATC1 


\section{Introduction}

Bone remodeling depends on the dynamic balance and precise coordination of bone resorption and subsequent bone formation, which are driven by osteoclast and osteoblast activation, respectively. Disturbances of this delicate balance lead to skeletal diseases, such as osteopenia, osteoporosis and osteopetrosis (Boyle et al, 2003). Bone-resorbing osteoclasts are multinucleated cells derived from monocyte-macrophage precursors which originated from hematopoietic stem cells, whereas bone-forming osteoblasts are derived from mesenchymal stem cells (MSC)(Boyle et al, 2003; Dennis et al, 1999; Pittenger et al, 1999; Teitelbaum, 2000). Osteoblast lineage cells produce RANKL and stimulate their RANK receptors on osteoclast precursors, resulting in osteoclast differentiation by activating the downstream signaling pathways such as transcription factors NF- $\kappa \mathrm{B}$, c-Fos, and nuclear factor of activated T cells c1 (NFATc1)(Teitelbaum \& Ross, 2003). To initiate bone resorption, osteoclasts need to successfully assemble a typical adhesion structure that is called actin ring or sealing zone, which consists of a belt of densely packed podosomes interlinked by an acto-myosin network (Georgess et al, 2014; Touaitahuata et al, 2014).

Exacerbated by general population aging, osteoporosis has become a dominating health problem around the world (Sambrook \& Cooper, 2006). Various conditions can provoke osteoporosis, such as bone metastasis, disability and inflammation, furthermore, in postmenopausal women, estrogen deficiency gives rise to excessive bone loss.(Touaitahuata et al, 2016) Despite recent advances in bone biology, the precise molecular mechanisms responsible for pathological osteoporosis remain elusive. Therefore, clarifying the molecular mechanisms and novel molecules involved in the maintenance of bone homeostasis is critical for deeper understanding of skeletal health and development of novel therapeutics against various bone disorders.

Lipid kinases and their phosphoinositide products perform essential functions in secretory vesicle trafficking. Phosphoinositide (PI) contributes to numerous basic biological processes, such as chemotaxis, intercellular trafficking, polarity formation and cytokinesis. (Takenawa \& Itoh, 2001) PIs are essential not only as membrane 
components in Eukaryotes and as precursors of second messengers like IP3 and PIP3 but also act as specialized membrane docking sites for effectors of diverse signaling cascades.(Takenawa \& Itoh, 2001) Accumulating evidence indicates that PIs and PI-interacting proteins such as Rho, Arf, and Rab small GTPases serve as modulators of osteoclast differentiation.(Chellaiah, 2006; Ory et al, 2008) PIs, especially PIP2, PIP3 and IP3, have been reported to serve various essential roles during osteoclast differentiation and function as well as in maintain bone homeostasis. PIP5K1 $\beta$ is a member of the Type 1 phosphatidylinositol 4-phosphate 5-kinases (PIP5K1s; $\alpha, \beta$, and $\gamma$ ), which are a family of isoenzymes producing phosphatidylinositol 4, 5-bisphosphate [PI $(4,5) \mathrm{P} 2]$ using phosphatidylinositol 4-phosphate as substrate(De Matteis \& Godi, 2004; Ishihara et al, 1996; Ishihara et al, 1998; Kanaho et al, 2007; Oude Weernink et al, 2004; van den Bout \& Divecha, 2009). PIP5K1s have been reported to be involved in a wide range of cellular functions not only as an enzyme generating PIP2, which is a critical regulator of cell adhesion formation, actin dynamics, and membrane trafficking, but also as cell signaling modulation factor, such as cytoskeleton assembly, exocytosis, endocytosis, cell apoptosis, and so on(Mao \& Yin, 2007; van den Bout \& Divecha, 2009). PIP5k1 $\gamma$, which is a major PtdIns (4,5)P2-synthesising enzyme in the rodent brain with three splicing variants, is elegantly involved in neurons and neuroendocrine cells: Hara Y et al reported that PIP5K1 $\gamma$, particularly PIP5K1 $\gamma_{-} \mathrm{i} 2$, performs essential roles in neuronal migration, possibly through recruitment of adhesion components such as talin and focal adhesion kinase to the plasma membrane.(Hara et al, 2013) In murine megakaryocytes, PIP5K1 $\gamma$ defect results in plasma membrane blebbing accompanied by a decreased connection of the membrane to the cytoskeleton possibly through a pathway involving talin.(Wang et al, 2008b) Moreover, PIP5K1 $\gamma_{-}$i2 revealed unique regulatory and targeting mechanisms through its $\mathrm{C}$-terminal 26 amino acids. In neurons, PIP5K1 $\gamma_{-}$i2 modulates the clathrin-mediated endocytosis of synaptic vesicles at presynapses and $\alpha$-amino-3-hydroxy-5-methyl-4-isoxazolepropionate-type glutamate receptors during long-term depression at postsynapses via association with adaptor protein complex (AP)-2(Unoki et al, 2012). In non-neuronal cells, PIP5K1 $\gamma_{-}$i2 also regulates the formation of focal adhesions through the interaction with talin.(Di Paolo 
et al, 2002; Ling et al, 2002; Sun et al, 2007) Furthermore, targeted disruption of PIP5K1 $\gamma$ leads to widespread developmental and cellular defects. PIP5K1 $\gamma$-null embryos have myocardial developmental defects including impaired intracellular junctions resulting in heart failure and extensive lethality at embryonic day 11.5 as well as impaired PIP2 production, adhesion junction formation, and neuronal cell migration that lead to neural tube closure defects.(Wang et al, 2007) PIP5k1 $\alpha$, was reported to selectively modulate apical endocytosis in polarized renal epithelial cells and to regulate invadopodia formation and ECM degradation in human breast cancer cells by localized production of $\mathrm{PI}(4,5) \mathrm{P}(2)$ (Szalinski et al, 2013). The function of PIP5k1 $\beta$ was investigated less and the roles of PIP5k1s in maintaining bone homeostasis were rarely reported. Only one report indicated that PIP5k1 $\gamma$ deficiency or overexpression delayed osteoclast differentiation and excess of PIP5k1 $\gamma$ disrupted osteoclasts cytoskeleton in a talin-independent way.(Zhu et al, 2013)

In the present study, we found that PIP5k1 $\beta$ was highly expressed during RANKL-induced osteoclast differentiation and PIP5k1 $\beta$ deletion in mice resulted in bone loss. To further understand the role of PIP5k1 $\beta$ in bone homeostasis, we investigated the functions of PIP5k1 $\beta$ acting on osteoclast and osteoblast differentiation by gain- and loss-of-function in vitro. We found that PIP5K $1 \beta$ can repress the proliferation and migration of bone-marrow-derived macrophage-like cells (BMMs) to inhibit osteoclast differentiation. Furthermore, PIP5k1 $\beta$ deletion promoted the activation of MAPK and Akt signaling cascades and enhanced expression of TRAF6 and c-Fos to facilitate NFATC1 expression and nuclear translocation, thereby accelerating osteoclast differentiation and function. Last but not the least, PIP5k1 $\beta$ deficiency upregulated Grb2 expression during osteoclast differentiation. PIP5K1 $\beta$ also enhanced osteoblast differentiation through activating of smad1/5/8. Thus, PIP5K1 $\beta$ can regulate bone mass and bone remodeling.

\section{Results}

\section{PIP5k1ß-deficient mice show an osteoporosis bone phenotype.}

Having demonstrated that PIP5k1 $\gamma$ served an essential role in modulating 
osteoclast differentiation and that it must be expressed at an actually exact level to maintain normal differentiation of osteoclast.(Zhu et al, 2013) To determine the role of PIP5k1 $\beta$ in the skeleton, we evaluated the mutant mouse strain with piggyback (PB) transposition system-induced mutation in the PIP5k1 $\beta$ gene. Homozygous mutation of PIP5k1 $\beta$ were born healthy at the predicted Mendelian frequencies. PIP5k $1 \beta^{-/-}$mice had a similar body structure to that of WT littermates with no obvious differences observed between PIP5k1 $\beta^{-/-}$and WT controls (Supplementary Fig. 1A). Interestingly, micro computed tomography (micro CT) analysis revealed that the trabecular bone mass density (BMD), trabecular bone volume versus tissue volume (BV/TV), trabecular bone number (Tb.N.) and trabecular bone thickness (Tb.Th.) were significantly lower but trabecular bone separation (Tb.Sp.) is obvious high in PIP5k1 $\beta^{-/-}$mice compared to that of age- and sex-matched WT littermates (Fig. 1A-F), suggesting defects in trabecular bone growth. To gain further insight into the in vivo cellular phenotype of the PIP5k1 $\beta^{-/}$mice, bone histomorphometry was performed on decalcified sections stained for TRAP activity and for hematoxylin and eosin staining (Fig. 1G). Consistent with micro CT data, histomorphometric analysis of tibia from 8-week-old PIP5k1 $\beta^{-1-}$ mice revealed a lower trabecular bone mass when compared to WT mice (Fig. 1G, H). Analysis of osteoclast parameters using TRAP stained sections showed that PIP5k1 $\beta^{-/}$mice exhibited a significant increase in the number of osteoclasts (Fig. 1G, I). Moreover, Dynamic histomorphometric analysis detected the reduced bone formation rate and mineral apposition rate in PIP5k1 $\beta^{-/-}$mice (Fig. 1J, K). Furthermore, the serum levels of CTX-1, a marker for bone resorption, was significantly increased (Fig. 1L) but serum OCN levels, a marker for bone formation, was dramatically decreased (Fig. 1O) in PIP5k1 $\beta^{-/}$mice. Moreover, immunohistochemistry detected that expression of CTSK, one of major markers of mature osteoclasts, in PIP5k1 $\beta$ deletion mice was significantly higher than that in wide type mice (Fig. 1M-N). These accumulating results suggest that PIP5k1 $\beta$ deletion leads to significant decrease in bone mass, which is possibly due to imbalance changes in osteoclasts bone resorption and osteoblasts bone formation.

PIP5k1及 expression was increased during RANKL-induced osteoclast 


\section{differentiation.}

Thereby, to determine how PIP5k1 $\beta$ influences osteoclasts formation and function, we first examined the transcriptomes of preosteoclasts and mature osteoclasts differentiated from mouse bone marrow-derived macrophage-like cells (BMMs) using cDNA microarray and found that among PIP5k1s only PIP5k1 $\beta$ was highly expressed in mature osteoclasts.(Fig. 2A). When we cultured BMMs in the presence of RANKL and M-CSF, the expression of PIP5k1 $\beta$ was markedly increased during RANKL-induced osteoclast differentiation since the third day of RANKL induction, however, the expressions of PIP5k1 $\alpha$ and PIP5k1 $\gamma$ were not changed (Fig. 2B, Fig.3B). Expression levels of VATPased2 and TRAP, important markers of osteoclastogenesis, increased as the cells differentiated (Fig. 2B). To confirm the importance of PIP5k1 $\beta$ in skeletal biology, we explored the expression pattern of PIP5k1 $\beta$ in different mice organs such as bone, spleen, brain, thymus, lung, kidney, liver, heart, and muscle and found that PIP5k1 $\beta$ was highly expressed in heart, lung, brain and bone (Supplementary Fig. 1B), which indicated that PIP5k1 $\beta$ might play a crucial role in bone biology. These results indicate that PIP5k1 $\beta$ might be involved in regulation of osteoclastogenesis and bone remodeling.

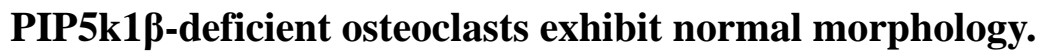

Accumulating evidences demonstrated that PIP5k1 family kinase serve central roles in cytoskeleton assembly(Kisseleva et al, 2005; Rozelle et al, 2000; Shibasaki et al, 1997; van den Bout \& Divecha, 2009; van Horck et al, 2002; Wang et al, 2008b), so we give rise to the hypothesis that PIP5k1 $\beta$ deficient may affect osteoclast cytoskeleton. To verify this hypothesis, we detect the actin ring formation as well as the localization of vinculin and Rac1, which are critical factors that can contribute to the ability of osteoclasts to rearrange podosomes into the sealing zone and establish the bone-resorbing apparatus, through classical confocal microscopy(Croke et al, 2011; Fukunaga et al, 2014; Ory et al, 2000; Sun et al, 2005; Wang et al, 2008a). F-actin exhibits a similar well-defined peripheral belt architecture in both WT and PIP5k1 $\beta$-deficient osteoclast, and PIP5k1 $\beta$ deletion promotes podosome formation in preosteoclasts and PIP5k1 $\beta$ deletion osteoclasts display much larger actin rings 
compared with WT osteoclasts. Vinculin and Rac1 localization and expression are also normal in PIP5k1 $\beta$-deletion osteoclasts, which are all enriched at the podosome belt and are colocalized with F-actin (Touaitahuata et al, 2016) (Figure. 2C). Finally, the expression levels of $\beta 3$ integrin, which involves in the association of osteoclasts with bone matrix to trigger osteoclast bone resorption activity, was also uninfluenced by PIP5k1 $\beta$ deficiency (Supplementary Fig. 1C, D).These results indicate that PIP5k1 $\beta$ deletion accelerates actin ring formation but does not affect osteoclast cytoskeleton assembly and sealing zone formation as well as interaction ability with bone matrix on the whole.

\section{Deficiency of PIP5k1ß enhanced osteoclast differentiation and bone} resorption. Given our observation that PIP5k1 $\beta$ expression is upregulated during RANKL-induced oseoclastogenesis, we examined the effect of PIP5k1 $\beta$ on osteoclast differentiation. Bone marrow cells from 9-week- old WT and PIP5k1 $\beta^{-/-}$mice were cultured with M-CSF to generate WT and PIP5k1 $\beta^{-/-}$BMMs, which were used as osteoclast precursor cells to be stimulated with RANKL and M-CSF. Deletion of PIP5k1 $\beta$ in BMMs significantly stimulates the formation of TRAP-positive multinucleated cells (TRAP-positive MNCs) mediated by RANKL (Fig. 3A, C). More osteoclasts with 3-8 nuclei or >13 nuclei were observed in the PIP5k1 $\beta$-deficient group. Besides, RT-PCR results exhibited an increased expression of DC-STAMP in PIP5k1 $\beta^{-/-}$BMMs compared with WT BMMs during RANKL-induced osteoclast differentiation (Fig. 3B). Consistently, gene expression analysis revealed that PIP5k1 $\beta$ expression was significantly increased since the third day of RANKL-induced osteoclastogenesis, but the expressions of the other two PIP5k1 family proteins PIP5k1 $\alpha$ and PIP5k1 $\gamma$ were not obviously changed during both WT and PIP5k1 $\beta$ deletion BMMs differentiation into osteoclasts (Fig. 3B, D). Moreover, the expressions of the marker genes of osteoclast formation, such as TRAP, CTSK, ATPasev0d2, NFATC1, DC-STAMP and OSCAR were dramatically enhanced during PIP5k1 $\beta$ deficiency BMMs differentiation into osteoclasts compared with that of WT BMMs. (Fig. 3E-J) Furthermore, we cultured WT and PIP5k1 $\beta^{-/-}$BMMs on bovine bone and initiated osteoclastogenensis by M-CSF and RANKL stimulation, 
respectively. After successful formation of mature osteoclasts, the cells were cultured for another day with M-CSF and RANKL stimulation to further resorb bone; the resorbed bones then underwent SEM. We discovered that PIP5k1 $\beta$ deletion promoted osteoclasts bone resorption and the resorption area of the bone slices by PIP5k1 $1 \beta^{-/}$ osteoclasts was significantly larger than that of WT osteoclasts. (Fig. $3 \mathrm{~K}-\mathrm{L}$ ) These results suggest that PIP5k1 $\beta$ deficiency enhances osteoclast differentiation and formation as well as promotes osteoclasts bone resorption function; and this function might not depend on the compensational role of PIP5k1 $\alpha$ and PIP5k1 $\gamma$.

\section{Overexpression of PIP5k1 $\beta$ arrests osteoclastogenesis.}

To further verify the role of PIP5k1 $\beta$ in osteoclast differentiation and formation, we overexpress PIP5k1 $\beta$ in BMMs to evaluate its effect on RANKL-induced osteoclast differentiation. PIP5k1 $\beta$ was overexpressed in the BMMs by lentivirus transfection into the BMM cells and then the BMMs were cultured with RANKL and M-CSF. The overexpression levels of PIP5k1 $\beta$ with gradually increased dose of lentivirus were showed by RT-PCR (Fig. 4A). TRAP staining analysis showed that overexpression of PIP5k1 $\beta$ significantly inhibits osteoclast differentiation and the inhibition effect was gradually increased along with the increasing dose of PIP5k1 $\beta$ overexpression. (Fig. 4 B-E)

\section{Deficiency of PIP5k1 $\beta$ enhances proliferation, migration and NFATc1 nuclear localization in preosteoclasts.}

To explore the mechanisms of PIP5k1 $\beta$ inhibition of osteoclast differentiation, we examined the effect of PIP5k1 $\beta$ on BMMs proliferation and migration. The wound healing assay displayed that PIP5k1 $\beta$ deletion promotes preosteoclasts migration triggered not only by M-CSF or by both M-CSF and RANKL (Fig. 5A, B). The CCK8 assay showed that PIP5k1 $\beta$ deficiency enhanced BMMs proliferation under the induction of RANKL and M-CSF, whereas only RANKL or M-CSF had no significant effect on the proliferation of BMMs (Fig. 5C). Furthermore, PIP5k1 $\beta$ deficiency facilitated NFATC1 expression and nuclear translocalization when triggered by RANKL detected by classical confocal microscopy and western blotting (Fig. 5D-E). Besides, luciferase assay with a NFATC1 responsive reporter was strongly dampened 
by PIP5k1 $\beta$ overexpression under the stimulation of RANKL (Fig. 5F-G). These results implied that PIP5k1 $\beta$ suppressed osteoclast differentiation by depressing preosteoclast proliferation, migration and also reducing NFATC1 signaling by decreasing its expression and nuclear translocalization.

Deficiency of PIP5k1 $\beta$ leads to increases of M-CSF- and RANKL-mediated MAPK, c-Fos and AKT signaling cascades.

PIP5k1 $\beta$ was discovered to be highly expressed during RANKL-mediated osteoclastogenesis and PIP5k1 $\beta$ suppressed osteoclast formation and bone resorption capacity. We asked which signaling pathway modulated osteoclast differentiation and whose function is affected by the absence of PIP5K1 $\beta$, which in turn affected osteoclastogenesis. First, we examined the archetypical RANKL signaling mediators such as c-Fos, TRAF6, NFATC1, Cathepsin K (CTSK) and TRAP, and found that deletion of PIP5k1 $\beta$ prominently enhanced the expression of these genes during RANKL-mediated osteoclstogenesis (Fig. 3E-F, H, Fig. 6A-D). During RANKL-induced osteoclastogenesis, ligation of RANKL with its receptors RANK leads to recruitment of TRAF6 by RANK, which leads to activation of nuclear factorKB (NF-KB), the mitogen-activated kinases (MAPKs) and the Akt pathways.(Kadono et al, 2005; Kobayashi et al, 2001) In this context, we detected which of these pathways were modulated by PIP5k1 $\beta$. The RANKL-induced activating phosphorylation of Akt and three typical MAP kinases (p38, ERK1/2 and JNK) were substantially elevated in PIP5k1 $\beta$ deletion BMMs compared with that of WT BMMs (Fig. 6E-J).

Then, we treated WT and PIP5KI $\beta^{-/}$mice osteoclast precursors with M-CSF and assessed activation of established effector molecules, which mediated osteoclast formation. Absence of PIP5K1 $\beta$ had certain effects on M-CSF-induced phosphorylation of ERK, JNK or AKT in triggering with M-CSF but the enhance effects were not consistent along with the time of cells exposed to M-CSF, especially for JNK and ERK1/2, compared with WT BMMs (Fig.6K-N). Notably, the expressions of Grb2 was also enhanced during RANKL-mediated osteoclastogenesis in PIP5k1 $\beta^{-/-}$osteoclasts compared with WT osteoclasts (Fig.6A). The adaptor protein 
Grb2 has been reported to play a pivotal role in several tyrosine kinase signal transduction pathways and previous study demonstrated that Grb2 promotes osteoclasts survival through activating Erk activation and bone resorption activity by enhancing their adhesion.(Levy-Apter et al, 2014) These data suggested that PIP5k1 $\beta$ deficiency influenced M-CSF or RANKL-induced osteoclastogenic properties to a certain extent, particularly RANKL-induced c-Fos, MAPK and AKT signaling pathways. Taken together, PIP5k1 $\beta$ suppressed osteoclasts differentiation and function might through influencing M-CSF- and RANKL-mediated MAPK, c-Fos and AKT signaling cascades.

\section{PIP5k1 $\beta$ accelerates osteoblast differentiation.}

Given that the serum bone formation marker OCN was significantly decreased in PIP5k1 $\beta^{-/-}$mice compared with WT mice, we explored the effect of PIP5k1 $\beta$ on osteoblast differentiation and function to deepen our understanding of the role of PIP5k1 $\beta$ in bone homeostasis. First, immunohistochemistry revealed that expression of OSX, one of major transcriptional factors that control osteoblast differentiation, in PIP5k1 $\beta$ deletion mice was notably lower than that in wide type mice (Fig. 7A-B). Then, BMSCs constantly overexpress or knockdown of PIP5k1 $\beta$ were obtained by corresponding lentivirus transfection (Fig. 7C) and underwent osteoblast differentiation by stimulation with osteogenic medium. MTT assay revealed that knockdown or overexpression of PIP5k1 $\beta$ have no effect on BMSCs proliferation (Supplementary Fig.2A). Real-time PCR was performed to determine whether osteoblast marker genes were differentially expressed between WT and PIP5k1 $\beta$ overexpression osteoblasts. The mRNA levels of PIP5k1 $\beta$ were significantly enhanced during osteoblast differentiation and the mRNA expression of osteoblast marker genes, ALP, SP7, colla1, OCN and BSP, were increased by overexpression of PIP5k1 $\beta$ (Fig. 7D-K). Increased ALP activity was observed in PIP5k1 $\beta$-overexpression osteoblasts at day 7 of culture and decreased ALP activity was observed in PIP5k1 $\beta$-knockdown osteoblasts at day 7 of culture as compared to WT osteoblasts by ALP staining (Fig. 7L). The bone mineralization activity of osteoblasts derived by BMSCs constantly overexpression or knockdown of PIP5k1 $\beta$ was also 
altered consistently with ALP activity alteration relative to WT osteoblasts, as determined by alizarin red S staining and analysis at day 14 of culture (Fig. 7M). Furthermore, the protein levels of Col1 $\alpha 1$, RUNX2 and OSX, which were central marker genes of osteoblastogenesis were all enhanced via PIP5k1 $\beta$ overexpression and decreased when PIP5k1 $\beta$ was knockdown. (Fig. 7N, supplementary Fig. 2B-C). These results implied that PIP5k1 $\beta$ enhanced MSCs differentiation into osteoblast.

PIP5k1及 enhances osteoblast differentiation through activating p-Smad1/5/8 signaling.

Several signaling pathways were involved in regulation of BMSCs differentiation into osteoblast, of which smad1/5/8 signaling exerts crucial roles in osteogenesis. To understand through which pathways PIP5k1 $\beta$ modulates osteoblast differentiation, we tested the activation of main pathways that regulates MSCs differentiation into osteoblast including smad1/5/8 and $\beta$-catenin and found that PIP5k1 $\beta$ prominently modulates smad1/5/8 activation. The phosphorylation of smad1/5/8 was significantly increased after 7 and 14 days treatment with osteogenic medium compared with cells treated with control medium, while, knockdown of PIP5k1 $\beta$ drastically depressed smad1/5/8 phosphorylation but overexpression of PIP5k1 $\beta$ outstandingly increased its phosphorylation on the contrary (Fig. 8A-B). To refine this finding, we further treated BMSCs with LDN193189, a smad1/5/8 specific inhibitor, to eliminate the activation of smad1/5/8. We observed that PIP5k1 $\beta$ overexpression prominently enhanced osteoblast differentiation and LDN193189 significantly reduced osteogenesis, but the reduction ratio was partly rescued by overexpression of PIP5k1 3 (Fig. 8C-E). Western blotting showed that PIP5k1 $\beta$ overexpression violently enhanced osteoblastogenesis master genes, Col1a1, Runx2 and OSX, expression, and LDN193189 significantly inhibited expression of these genes, but the inhibition ratio was partly rescued by overexpression of PIP5k1 $\beta$. Meanwhile, Phosphorylation of smad1/5/8 was changed in consistent with the change trend of these osteoblastogenesis master genes. (Fig. 8F). In summary, these observations indicated that PIP5k1 $\beta$ enhances BMSCs differentiation into osteoblast partly through activating smad1/5/8 signaling.

\section{Discussion}


Several therapeutic strategies have been developed for excess bone loss diseases such as osteoporosis, including direct suppression of osteoclasts resorption capacity or irritation of osteoblast bone formation, with compromised efficacy and momentous side effects. Therefore, more appropriate treatments need to be explored to synchronously modulate the interaction between osteoblasts and osteoclasts. In present study, we discovered that PIP5K1 $\beta$ inhibited osteoclasts formation and function but meanwhile facilitated osteoblast differentiation, so that PIP5k1 $\beta^{-/-}$displayed obvious osteoporosis phenotype. These hinted that PIP5K1 $\beta$ might serve pivotal roles in maintaining bone homeostasis and that deepening understanding of the mechanisms of its functional role might provide new strategy for the prevention and treatment of osteoporosis.

Among all the family members of PIP5k1 family kinases that generate PIP2, only PIP5k1 $\beta$ was highly expressed in bone and during osteoblastogenesis and RANKL-induced osteoclast differentiation, which indicated that PIP5k1 $\beta$ might serve crucial roles in bone biology. Under the consideration that PIP5k1 family proteins were reported to be involved in cytoskeleton assembly and that the podosome reorganization of osteoclasts was a major event for mature osteoclasts formation and activity(Mao \& Yin, 2007; Teitelbaum, 2011; van den Bout \& Divecha, 2009), we speculated that PIP5k1 $\beta$ might participate in osteoclast sealing zone organization. However, our present study showed that PIP5k1 $\beta$ deletion osteoclasts exhibit no apparent cytoskeletal abnormalities and also had no impact on the expression and colocalization of proteins involved in sealing zone formation, such as vinculin, Rac1 and F-actin. Additionally, the expression levels of $\beta 3$ integrin, which takes part in the association of osteoclasts with bone matrix to trigger the osteoclast bone resorption activity, was also uninfluenced by PIP5k1 $\beta$ deficiency (Supplementary Fig. 1C, D). This accumulating evidence indicated that PIP5k1 $\beta$ affects osteoclasts not by affecting their cytoskeleton formation and interaction with bone matrix though deletion of PIP5k1 $\beta$ accelerates and enlarges osteoclast actin ring formation. Nonetheless, PIP5k1 $\beta$ indeed influences osteoclast formation and function, which might be buttressed by the following facts. First, PIP5k1 $\beta$ deletion enhanced the 
expression of osteoclasts marker genes such as ACP5, CTSK, DC-STAMP, c-Fos, NFATC1 and so on. Besides, PIP5k1 $\beta$ deficiency facilitated osteoclast bone resorption as proven by enhanced pit formation activity and highly elevated serum level of bone resorption marker CTX-1 in PIP5k1 $\beta$ deletion mice. Finally, knockdown and overexpression of PIP5k1 $\beta$ in BMMs accelerated and depressed osteoclast differentiation, respectively, as detected by TRAP staining.

Osteoclasts are generated from mononuclear hematopoietic myeloid lineage cells, which are derived in the bone marrow and are attracted to the blood stream by factors. These circulating precursors migrated to bone surfaces undergoing resorption by chemokines and other factors that were modulated by M-CSF and RANKL at these sites, where they fuse to develop multinucleated bone resorbing cells(Kikuta et al, 2011; Kular et al, 2012). Interestingly, in the present study, we obtained that PIP5k1 $\beta$ deletion can accelerate preosteoclast migration triggered by M-CSF or by both M-CSF and RANKL. Besides, PIP5k1 $\beta$ deletion promoted preosteoclast proliferation. These results indicated that PIP5k1 $\beta$ repressed preosteoclast proliferation and migration to inhibit osteoclastogenesis, but the molecular mechanisms remain to be explored.

Furthermore, our present study suggest that PIP5k1 $\beta$ affected osteoclast differentiation and function also through modulation of NFATC1 which represents a master switch that modulates terminal differentiation of osteoclasts downstream of RANKL(Asagiri et al, 2005; Takayanagi et al, 2002), as supported by several discoveries. First, we found that PIP5k1 $\beta$ deficiency enhanced NFATC1 expression and facilitated NFATC1 nuclear translocation. Additionally, overexpression of PIP5k1 $\beta$ dampened NFATC1 activity. Moreover, the phosphorylation of Akt and the activation of MAPK/p38, ERK1/2 and JNK, which mediated NFATC1 activation during RANKL-stimulated osteoclast differentiation, were prolonged and enhanced with RANKL stimulation in PIP5k1 $\beta^{-/-}$osteoclasts. Last but not the least, PIP5k1 $\beta$ deletion enhanced TRAF6 and c-Fos expression, which were demonstrated to stimulate the downstream mediators to trigger expression and nuclear translocalization of NFATC1 resulting in enhanced osteoclastogenesis.(Boyce \& Xing, 2008; Kadono et al, 2005) (Wang et al, 1992) PIP5K1 $\gamma$ was reported to modulate calcium transport, which in turn, 
initiated NFATc1 (Wang et al, 2004). Moreover, NFATC1 can autoamplify and trigger calcitonin receptor expression which eventually upregulated calcium signaling (Asagiri et al, 2005). Further studies need to be performed to determine if PIP5k1 $\beta$ deletion affects calcium signaling.

Adaptor protein Grb2 (growth-factor-receptor-bound protein 2), which serves an important role in several tyrosine kinase signal transduction pathways, was reported to play a crucial role in osteoclastogenesis by increasing Erk and Akt signaling motivated by M-CSF to promote osteoclast proliferation, differentiation and survival.(Levy-Apter et al, 2014; Ross \& Teitelbaum, 2005; Takayanagi, 2007) An amusing result was discovered in the present study that Grb2 expression was advanced and dramatically up-regulated during osteoclastogenesis while PIP5k1 $\beta$ was deleted, besides, the ERK1/2 signaling was also enhanced. These implied that PIP5k1 $\beta$ might modulate Grb2 expression to regulate osteoclastogenesis. However, further study is needed to make deep insight in the association between PIP5k1 $\beta$ and Grb2 expression.

Our present work also discovered that PIP5k1 $\beta$ facilitated osteoblast differentiation, immunohistochemistry in Fig. 7A showed that $\mathrm{OSX}^{+}$cells was prominently decreased both in bone marrow cells and in bone lining cells in PIP5k1 $\beta$ deletion mice compared with that of WT mice, which indicated that PIP5k1 $\beta$ might stimulate MSCs differentiation into osteoblast through up-regulation of OSX expression. Furthermore, the expression of osteoblast differentiation early and late markers Col1 $\alpha 1$, ALP, OCN as well as BSP and osteoblast differentiation master transcription factor Runx2 and OSX were all outstandingly elevated or reduced during osteoblastogenesis of PIP5k1 $\beta$ overexpression or knockdown BMSCs respectively. Moreover, ALP and Alizarin Red staining demonstrated that overexpression or knockdown of PIP5k1 $\beta$ promoted or repressed MSCs differentiation into osteoblast and calcium precipitation, respectively. Meanwhile, the phosphorylation of smad1/5/8 was also enhanced or dampened in accordance with overexpression or knockdown of PIP5k1 $\beta$ during BMSCs differentiation into osteoblast. Moreover, PIP5k1 $\beta$ overexpression can partly rescue the elimination effect of osteoblast differentiation and smad1/5/8 activation upon LDN193189, a smad1/5/8 specific inhibitor, administration. 
These accumulating evidences implied that PIP5k1 $\beta$ facilitates osteoblast differentiation from BMSCs partly through smad1/5/8 signaling.

In summary, our study explored the role of PIP5k1 $\beta$ in bone biology, particularly in osteoclast and osteoblast differentiation and function, for the first time. We found that PIP5K1 $\beta$ was highly expressed both during osteoclast and osteoblast differentiation and PIP5K1 $\beta$ can regulate bone mass and bone remodeling by inhibiting osteoclast differentiation and facilitating osteoblast differentiation. (Supplementary Fig. 2D) Further studies need to be performed to determine if these functions were partly caused by PIP2 levels alteration that was contributed by PIP5k1 $\beta$ kinase activity during osteoclast and osteoblast differentiation. Besides, further explanation of the RANKL modulation of PIP5k1 $\beta$ expression is needed to deepen our understanding of the biological roles of PIP5k1 $\beta$ on maintaining bone homeostasis to explore new targets for prevention and treatment of bone disorders.

\section{Materials and Methods}

Reagents. Alpha-minimum essential medium (MEM), fetal bovine serum (FBS), and penicillin were purchased from Gibco BRL (Gaithersburg, MD, USA). Recombinant mouse macrophage-colony stimulating factor (M-CSF) was purchased from R\&D Systems (USA). Recombinant Murine sRANK Ligand was purchased from Peprotech (USA).Tartrate-resistant acid phosphatase (TRAP) staining solution was obtained from Sigmae-Aldrich (St. Louis, MO, USA). The Cell Counting Kit-8 (CCK-8) was obtained from Dojindo Molecular Technology (Japan).

Animals. FVB WT and PIP5k1 $\beta^{-/}$mice were obtained from PBmice of Fudan University (http://www.idmshanghai.cn/PBmice or http://www.scbit.org/PBmice/) and housed five per cage under standard conditions $\left(12 \mathrm{~h}\right.$ light $/ 12 \mathrm{~h}$ dark cycle, $21^{\circ} \mathrm{C}$ controlled temperature).

Sample preparation and skeletal morphology. For microCT analysis, right femurs of both WT and PIP5k1 $\beta^{\top} /$ mice were fixed with $4 \%$ paraformaldehyde and analyzed by Scanco Medical CT-40 instruments. Trabecular bone analysis was performed on the secondary spongiosa region $(300 \mu \mathrm{m}$ below the growth plate with a 
total height of $1 \mathrm{~mm}$ towards the mid shaft) of the distal femur. 3D images were generated in CTvol program (Skyscan). For histochemistry, proximal tibias from both WT and PIP5k1 $\beta^{-/-}$mice were fixed in $4 \%$ paraformaldehyde and embedded in paraffin, then cut longitudinally into 4-mm-thick sections and processed for hematoxylin and eosin staining (H\&E staining) or Tartrate-resistant acid phosphatase (TRAP) staining after decalcifying. To evaluate bone formation rate in vivo, WT and PIP5k1 $\beta^{-/-}$mice were injected with green fluorescent calcein (Sigma-Aldrich; $5 \mathrm{mg}$ per kg body weight) on 8 and 2 days before euthanasia. Then the tibiae were dissected and embedded in methyl methacrylate resins. Tissue sections were observed under a laser-scanning microscope (LSM5 PASCAL; Carl Zeiss)

Cell Culture. WT or PIP5k1 $\beta^{-/}$BMMs were obtained as described previously. (Li et al, 2013; Qin et al, 2012)In brief, bone marrow cells extracted from the femurs and tibiae of a 9- to 10-week-old WT or its littermate PIP5k1 $\beta^{-/-}$mouse were cultured in $\alpha$-MEM medium containing $15 \mathrm{ng} / \mathrm{ml}$ M-CSF (416-ML ; R\&D; USA) in a T-75 $\mathrm{cm}^{2}$ flask for proliferation for 2-3 days until reaching $90 \%$ confluence, the cells were washed with PBS three times and trypsinized for 30 min to harvest BMMs. Adherent cells were classified as BMMs. WT and PIP5k1 $\beta^{-/-}$BMMs were plated on 96-well plates at a density of $8 \times 10^{3}$ cells/well in triplicate and incubated with $\alpha$-MEM medium containing $20 \mathrm{ng} / \mathrm{ml} \mathrm{M-CSF}$ in a humidified incubator containing $5 \% \mathrm{CO} 2$ at $37^{\circ} \mathrm{C}$ for $2 \mathrm{~d}$. Then, the cells were cultured with $\alpha$-MEM medium with M-CSF $(20 \mathrm{ng} / \mathrm{mL})$ and RANKL (75 ng /mL; 315-11; Peprotech; Rocky Hill, NJ) for indicated times. Moreover, the medium was changed every other day. For osteoblast differentiation, bone mesenchymal stem cells (BMSCs) were gained from 9- to 10-week-old mice and expanded as previously described, induced by culturing cells in osteogenic medium (DMEM containing $1 \mathrm{M} \beta$-glycerophosphate, 50mM ascorbic acid and 1mM Dex (methylisobutylxanthine)) for indicated times, followed by subsequent experiments.

TRAP activity assay. After 7 days of culture, the osteoclasts were fixed with 4\% paraformaldehyde (PFA) in PBS for $10 \mathrm{~min}$ and then rinsed three times with PBS, followed by TRAP staining, using an acid phosphatase kit (387A; Sigmae-Aldrich) according to the manufacturer's description and counter-stained with hematoxylin for 
$10 \mathrm{~s}$ to $30 \mathrm{~s}$. Images were obtained with a Nikon SMZ 1500 stereoscopic zoom microscope (Nikon Instruments Inc.; Melville, NY, USA). We randomly quantified the total area of TRAP-positive regions and the total number of osteoclasts on five selected fields of view for each sample.

Immunofluorescence. For F-actin ring immunofluorescent staining, WT and PIP5k1 $\beta^{-/-}$osteoclasts were fixed with $4 \%$ PFA for $15 \mathrm{~min}$ at room temperature and permeabilized for 5 min with $0.1 \%$ v/v Triton X-100 on ice. Cells were incubated with Fluorescent phalloidins (1:40; Invitrogen Life Technologies, USA) diluted in 1\% w/v bovine serum albumin-PBS for $20 \mathrm{~min}$ at room temperature and then washed extensively with PBS. Cells were then incubated with Hoechst 3342 dye (1:5000); Invitrogen Life Technologies, USA) for visualizing nuclei, washed with PBS, and mounted with ProLong Gold anti-fade mounting medium (Invitrogen Life Technologies, USA). Fluorescence was detected with NIKON A1Si spectral detector confocal system equipped with 20 (dry) lenses. Fluorescence images were obtained with NISeC Elements software. (National Institutes of Health).

Pit formation assay. For the bone resorption assay, $8 \times 10^{3}$ cells/well WT or PIP5k1 $\beta^{-/-}$BMMs were plated onto bovine bone slices in 96-well plates with three replicates and treated with M-CSF (20 ng/mL) and RANKL (75 ng/mL) for 8 days. Then the bone slices were fixed with $2.5 \%$ glutaraldehyde and followed by visualization with scanning electron microscope (SEM; FEI Quanta 250). Pit areas were quantified with Image $\mathbf{J}$ software (National Institutes of Health). Similar independent experiments were repeated three times.

Cell proliferation and migration assay. The proliferation rate was determined with CCK-8 assay according to the manufacturer's protocols. WT or PIP5k1 $1 \beta^{-/-}$BMMs were seeded in 96-well plates at a density of $8 \times 10^{3}$ cells/well, and incubated in complete a-MEM supplemented with $20 \mathrm{ng} / \mathrm{mL}$ M-CSF for $24 \mathrm{~h}$ until $60 \%$ confluences, then cells were treated with $20 \mathrm{ng} / \mathrm{ml} \mathrm{M-CSF}$ or $75 \mathrm{ng} / \mathrm{ml}$ RANKL or both for $24 \mathrm{~h}$. Then $100 \mathrm{ul} \mathrm{CCK}-8$ buffer was added to each well, followed by incubation at $37^{\circ} \mathrm{C}$ for an additional $2 \mathrm{~h}$. The absorbance was then measured at a wavelength of $450 \mathrm{~nm}(650$ nm reference) with an ELX800 absorbance microplate reader (Bio-Tek, USA). The 
migration ability was monitored with wound healing assay. In a typical procedure, a straight scratch was made gently through the monolayer cells using an Eppendorf tip in 48-well plates. Detached cells were washed away with PBS and the serum-free culture medium was added; cells were treated with $20 \mathrm{ng} / \mathrm{ml} \mathrm{M-CSF}$ or $75 \mathrm{ng} / \mathrm{ml}$ RANKL or both. Cells migrating to the scratch were monitored and images were taken at $0,17 \mathrm{~h}$ after wounding. The migration rate was calculated following the equation: percentage wound healing $=(($ wound length at $0 \mathrm{~h})-($ wound length at $17 \mathrm{~h})) /($ wound length at $0 \mathrm{~h})$ $\times 100$ (Davalos et al, 2012).

\section{RNA extraction and Q-PCR assay.}

WT and PIP5k1 $\beta^{-/-}$BMMs were plated in six-well plates at a density of $1 \times 10^{5}$ cells per well and incubated in complete a-MEM supplemented with $20 \mathrm{ng} / \mathrm{mL}$ M-CSF and $75 \mathrm{ng} / \mathrm{mL}$ RANKL for an indicated number of days. Total RNA was extracted with TRIzol reagent (Invitrogen) following the manufacturer's instructions and cDNA was generated with 1 ug of RNA. Then, real-time PCR was performed via the LightCycler480 system (Roche) using SYBR1Premix Ex TaqTM (Takara, Dalian, China) following manufacturer's instructions and data were analyzed using the comparison $\mathrm{Ct}\left(2^{-\Delta \Delta} \mathrm{Ct}\right)$ method The following program of real-time PCR was employed: denaturation at $95{ }^{\circ} \mathrm{C}$ for $10 \mathrm{~s}, 40$ cycles at $95{ }^{\circ} \mathrm{C}$ for $10 \mathrm{~s}$, and $60{ }^{\circ} \mathrm{C}$ for $30 \mathrm{~s}$. The dissociation stage was added to the end of the amplification procedure, and the dissociation curve did not show any nonspecific amplification. Glyceraldehyde-3-phosphate dehydrogenase (GAPDH) was used as housekeeping gene. All reactions were run in triplicate. The mouse primers used in the present study were shown as follows: GAPDH forward 5'- TTCACCACCATGGAGAAGGC -3' and reverse 5'- GGCATGGACTGTGGTCATGA-3'; Nfatc1 forward 5'-TCCGAGAATCGAGATCACCT-3' and reverse 5'-AGGGGTCTCTGTAGGCTTCC-3'; Acp5 (acid phosphatase 5) forward 5'-CGTCTCTGCACAGATTGCAT-3' and reverse 5'-AACTGCTTTTTGAGCCAGGA-3'; cathepsin $\mathrm{K} \quad$ (CTSK) forward 5'-GGACCCATCTCTGTGTCCAT-3' and reverse 5'-CCGAGCCAAGAGAGCATATC-3'; DC-STAMP_dendritic cell-specific 
transmembrane

protein

(DC-STAMP)

forward

$5^{\prime}-$

ACAAACAGTTCCAAAGCTTGC-3'

and

reverse

5'-TCCTTGGGTTCCTTGCTTC-3';

OSCAR

forward

5'-CTGCTGGTAACGGATCAGCTCCCCAGA-3'

and

reverse

5'-CCAAGGAGCCAGAACCTTCGAAACT-3';

PIP5k1 $\beta$

forward

$5^{\prime}-$

GAAGAAGCCCTGGGATCCCGACA-3'

and

reverse

5'-GGGTGTTTGGCTCAGCCGTCA-3';

ATP6v0d2

forward

$5{ }^{\prime}-$

AGACCACGGACTATGGCAAC-3'

and

reverse

5'-CAGTGGGTGACACTTGGCTA-3'

\section{Western blotting}

Cells were lysed on ice for 30 min with RIPA lysis buffer which contains $50 \mathrm{mM}$ Tris- $\mathrm{HCl}, \mathrm{pH}$ 7.4, $150 \mathrm{mM} \mathrm{NaCl}, 1 \%$ Nonidet P-40, and 0.1\% SDS supplemented with protease inhibitors $(10 \mathrm{mg} / \mathrm{ml}$ leupeptin, $10 \mathrm{mg} / \mathrm{ml}$ pepstatin A, and $10 \mathrm{mg} / \mathrm{ml}$ aprotinin). For Western blotting, $25 \mu \mathrm{g}$ of protein sample was resolved on $12.5 \%$ SDS-PAGE and electrotransferred onto nitrocellulose membranes (Whatman, Piscataway, NJ, USA). The primary antibodies used were as follows: phospho-Akt, total Akt, phosphor-GSK3 $\beta(\operatorname{ser} 9 A)$, PTEN, PLC $\gamma 2$ and all the primary antibodies involved in the MAPK signaling pathway (phospo-p44/42 ERK, total p44/42 ERK, phospho-p38, total-p38, and phospo-JNK and total-JNK) were purchased from Cell Signaling Technology (Danvers, MA, USA) and used at a 1:1000 dilution ratio. Anti-NFATC1 antibody was purchased from Santa Cruz and used at 1:500 dilution ratio. Beta-actin or GAPDH was used as loading control. HRP-conjugated secondary antibodies were used at a 1:5000 dilution. The antigen-antibody complexes were visualized using the enhanced chemiluminescence detection system (Millipore, Billerica, MA, USA) following the manufacturer's instructions. Immunoreactive bands were quantitatively analyzed in triplicate by normalizing the band intensities to their respective controls on scanned films using ImageJ software.

Alkaline phosphatase staining and Alizarin red staining. The cell layer was rinsed with PBS three times, followed by fixation in 4\% paraformaldehyde for $10 \mathrm{~min}$ at room temperature. Then cells underwent ALP or Alizarin red staining. ALP staining 
was performed according to the manufacturer's instructions. The fixed cells were then incubated with buffer containing $0.1 \%$ naphthol AS-Bi phosphate (Sigma-Aldrich) and $2 \%$ fast violet B (Sigma-Aldrich). After incubation for $1 \mathrm{~h}$ at $37{ }^{\circ} \mathrm{C}$, the cell layer was washed with deionized water. For Alizarin red staining, the fixed cells were rinsed with double-distilled $\mathrm{H} 2 \mathrm{O}(\mathrm{ddH} 2 \mathrm{O})$ and then stained with $40 \mathrm{mM}$ Alizarin red S (pH 4.9, Sigma) for $15 \mathrm{~min}$ with gentle agitation followed by five times washing with ddH2O.Then, the calcium precipitates were dissolved with $0.1 \mathrm{~N}$ sodium hydroxide and monitored using a Tecan Safire2 microplate reader (Tecan, Durham, NC, USA) by absorbance at $548 \mathrm{~nm}$ to quantify the degree of mineralization.

Statistical analysis. The data are presented as the mean \pm s.d. ( $\mathrm{n}$ is the number of tissue preparations, cells, or experimental replicates). For comparing groups of data, a two-tailed Student's $t$-test was used. A value of $\mathrm{P}<0.05$ was considered to be statistically significant.

\section{Author contributions}

Xiaoying Zhao: Conception and design, manuscript writing, collection of data, data analysis and interpretation; Guoli Hu and Chuandong Wang: Collection of data, Provision of study material; Lei Jiang and Jingyu Zhao: Collection of data and manuscript revision; Jiake Xu: Data analysis and manuscript revision; Xiaoling Zhang: Conception and design, manuscript revision, final approval of manuscript.

\section{Acknowledgments}

This work was supported by grants from National Natural Science Foundation of China (No.81572123, 81401844), Science and Technology Commission of Shanghai Municipality (No.14431900900, 15411951100, 16430723500), Shanghai Municipal

Education Commission-Gaofeng Clinical Medicine Grant Support (No.20161314) and Xin Hua Hospital Affilliated to Shanghai Jiao Tong University School of Medicine (009\&201601).

\section{Conflict of Interest}


The authors have declared that no conflict of interest exists.

\section{References}

Asagiri M, Sato K, Usami T, Ochi S, Nishina H, Yoshida H, Morita I, Wagner EF, Mak TW, Serfling E et al (2005) Autoamplification of NFATc1 expression determines its essential role in bone homeostasis. The Journal of experimental medicine 202: 1261-1269

Boyce BF, Xing LP (2008) Functions of RANKL/RANK/OPG in bone modeling and remodeling. Archives of biochemistry and biophysics 473: 139-146

Boyle WJ, Simonet WS, Lacey DL (2003) Osteoclast differentiation and activation. Nature 423: $337-342$

Chellaiah MA (2006) Regulation of podosomes by integrin alphavbeta3 and Rho GTPase-facilitated phosphoinositide signaling. European journal of cell biology 85: 311-317

Croke M, Ross FP, Korhonen M, Williams DA, Zou W, Teitelbaum SL (2011) Rac deletion in osteoclasts causes severe osteopetrosis. Journal of cell science 124: 3811-3821

Davalos V, Moutinho C, Villanueva A, Boque R, Silva P, Carneiro F, Esteller M (2012) Dynamic epigenetic regulation of the microRNA-200 family mediates epithelial and mesenchymal transitions in human tumorigenesis. Oncogene 31: 2062-2074

De Matteis MA, Godi A (2004) PI-loting membrane traffic. Nature cell biology 6: 487-492

Dennis JE, Merriam A, Awadallah A, Yoo JU, Johnstone B, Caplan Al (1999) A quadripotential mesenchymal progenitor cell isolated from the marrow of an adult mouse. Journal of bone and mineral research : the official journal of the American Society for Bone and Mineral Research 14: 700-709

Di Paolo G, Pellegrini L, Letinic K, Cestra G, Zoncu R, Voronov S, Chang S, Guo J, Wenk MR, De Camilli $P$ (2002) Recruitment and regulation of phosphatidylinositol phosphate kinase type 1 gamma by the FERM domain of talin. Nature 420: 85-89

Fukunaga T, Zou W, Warren JT, Teitelbaum SL (2014) Vinculin regulates osteoclast function. The Journal of biological chemistry 289: 13554-13564

Georgess D, Machuca-Gayet I, Blangy A, Jurdic P (2014) Podosome organization drives osteoclast-mediated bone resorption. Cell adhesion \& migration 8: 191-204

Hara Y, Fukaya M, Tamaki H, Sakagami H (2013) Type I phosphatidylinositol 4-phosphate 5-kinase gamma is required for neuronal migration in the mouse developing cerebral cortex. The European journal of neuroscience 38: 2659-2671 
Ishihara H, Shibasaki Y, Kizuki N, Katagiri H, Yazaki Y, Asano T, Oka Y (1996) Cloning of cDNAs encoding two isoforms of 68-kDa type I phosphatidylinositol-4-phosphate 5-kinase. The Journal of biological chemistry 271: 23611-23614

Ishihara H, Shibasaki Y, Kizuki N, Wada T, Yazaki Y, Asano T, Oka Y (1998) Type I phosphatidylinositol-4-phosphate 5-kinases. Cloning of the third isoform and deletion/substitution analysis of members of this novel lipid kinase family. The Journal of biological chemistry 273 : 8741-8748

Kadono Y, Okada F, Perchonock C, Jang HD, Lee SY, Kim N, Choi Y (2005) Strength of TRAF6 signalling determines osteoclastogenesis. EMBO reports 6: 171-176

Kanaho Y, Kobayashi-Nakano A, Yokozeki T (2007) The phosphoinositide kinase PIP5K that produces the versatile signaling phospholipid PI4,5P(2). Biological \& pharmaceutical bulletin 30: 1605-1609

Kikuta J, Iwai K, Saeki Y, Ishii M (2011) S1P-targeted therapy for elderly rheumatoid arthritis patients with osteoporosis. Rheumatology international 31: 967-969

Kisseleva M, Feng Y, Ward M, Song C, Anderson RA, Longmore GD (2005) The LIM protein Ajuba regulates phosphatidylinositol 4,5-bisphosphate levels in migrating cells through an interaction with and activation of PIPKI alpha. Molecular and cellular biology 25: 3956-3966

Kobayashi N, Kadono Y, Naito A, Matsumoto K, Yamamoto T, Tanaka S, Inoue J (2001) Segregation of TRAF6-mediated signaling pathways clarifies its role in osteoclastogenesis. The EMBO journal 20: $1271-1280$

Kular J, Tickner J, Chim SM, Xu J (2012) An overview of the regulation of bone remodelling at the cellular level. Clinical biochemistry 45: 863-873

Levy-Apter E, Finkelshtein E, Vemulapalli V, Li SS, Bedford MT, Elson A (2014) Adaptor protein GRB2 promotes Src tyrosine kinase activation and podosomal organization by protein-tyrosine phosphatase in osteoclasts. The Journal of biological chemistry 289: 36048-36058

Li H, Zhai Z, Liu G, Tang T, Lin Z, Zheng M, Qin A, Dai K (2013) Sanguinarine inhibits osteoclast formation and bone resorption via suppressing RANKL-induced activation of NF-kappaB and ERK signaling pathways. Biochemical and biophysical research communications 430: 951-956

Ling K, Doughman RL, Firestone AJ, Bunce MW, Anderson RA (2002) Type I gamma phosphatidylinositol phosphate kinase targets and regulates focal adhesions. Nature 420: 89-93

Mao YS, Yin HL (2007) Regulation of the actin cytoskeleton by phosphatidylinositol 4-phosphate 5 kinases. Pflugers Archiv : European journal of physiology 455: 5-18 
Ory S, Brazier H, Pawlak G, Blangy A (2008) Rho GTPases in osteoclasts: orchestrators of podosome arrangement. European journal of cell biology 87: 469-477

Ory S, Munari-Silem Y, Fort P, Jurdic P (2000) Rho and Rac exert antagonistic functions on spreading of macrophage-derived multinucleated cells and are not required for actin fiber formation. Journal of cell science 113 ( Pt 7): 1177-1188

Oude Weernink PA, Schmidt M, Jakobs KH (2004) Regulation and cellular roles of phosphoinositide 5-kinases. European journal of pharmacology 500: 87-99

Pittenger MF, Mackay AM, Beck SC, Jaiswal RK, Douglas R, Mosca JD, Moorman MA, Simonetti DW, Craig S, Marshak DR (1999) Multilineage potential of adult human mesenchymal stem cells. Science (New York, NY) 284: 143-147

Qin A, Cheng TS, Lin Z, Cao L, Chim SM, Pavlos NJ, Xu J, Zheng MH, Dai KR (2012) Prevention of wear particle-induced osteolysis by a novel V-ATPase inhibitor saliphenylhalamide through inhibition of osteoclast bone resorption. PloS one 7: e34132

Ross FP, Teitelbaum SL (2005) alphavbeta3 and macrophage colony-stimulating factor: partners in osteoclast biology. Immunological reviews 208: 88-105

Rozelle AL, Machesky LM, Yamamoto M, Driessens MH, Insall RH, Roth MG, Luby-Phelps K, Marriott G, Hall A, Yin HL (2000) Phosphatidylinositol 4,5-bisphosphate induces actin-based movement of raft-enriched vesicles through WASP-Arp2/3. Current biology : CB 10: 311-320

Sambrook P, Cooper C (2006) Osteoporosis. Lancet (London, England) 367: 2010-2018

Shibasaki Y, Ishihara H, Kizuki N, Asano T, Oka Y, Yazaki Y (1997) Massive actin polymerization induced by phosphatidylinositol-4-phosphate 5-kinase in vivo. The Journal of biological chemistry 272 : 7578-7581

Sun Y, Buki KG, Ettala O, Vaaraniemi JP, Vaananen HK (2005) Possible role of direct Rac1-Rab7 interaction in ruffled border formation of osteoclasts. The Journal of biological chemistry 280: 32356-32361

Sun Y, Ling K, Wagoner MP, Anderson RA (2007) Type I gamma phosphatidylinositol phosphate kinase is required for EGF-stimulated directional cell migration. The Journal of cell biology 178 : 297-308

Szalinski CM, Guerriero CJ, Ruiz WG, Docter BE, Rbaibi Y, Pastor-Soler NM, Apodaca G, Puthenveedu MA, Weisz OA (2013) PIP5KIbeta selectively modulates apical endocytosis in polarized renal epithelial cells. PloS one 8: e53790 
Takayanagi H (2007) Osteoimmunology: shared mechanisms and crosstalk between the immune and bone systems. Nature reviews Immunology 7: 292-304

Takayanagi H, Kim S, Koga T, Nishina H, Isshiki M, Yoshida H, Saiura A, Isobe M, Yokochi T, Inoue J et al (2002) Induction and activation of the transcription factor NFATc1 (NFAT2) integrate RANKL signaling in terminal differentiation of osteoclasts. Developmental cell 3: 889-901

Takenawa T, Itoh T (2001) Phosphoinositides, key molecules for regulation of actin cytoskeletal organization and membrane traffic from the plasma membrane. Biochimica et biophysica acta 1533: 190-206

Teitelbaum SL (2000) Bone resorption by osteoclasts. Science (New York, NY) 289: 1504-1508

Teitelbaum SL (2011) The osteoclast and its unique cytoskeleton. Annals of the New York Academy of Sciences 1240: 14-17

Teitelbaum SL, Ross FP (2003) Genetic regulation of osteoclast development and function. Nature reviews Genetics 4: 638-649

Touaitahuata H, Blangy A, Vives V (2014) Modulation of osteoclast differentiation and bone resorption by Rho GTPases. Small GTPases 5: e28119

Touaitahuata H, Morel A, Urbach S, Mateos-Langerak J, de Rossi S, Blangy A (2016) Tensin 3 is a new partner of Dock5 that controls osteoclast podosome organization and activity. Journal of cell science 129: 3449-3461

Unoki T, Matsuda S, Kakegawa W, Van NT, Kohda K, Suzuki A, Funakoshi Y, Hasegawa H, Yuzaki M, Kanaho Y (2012) NMDA receptor-mediated PIP5K activation to produce $\mathrm{PI}(4,5) \mathrm{P}(2)$ is essential for AMPA receptor endocytosis during LTD. Neuron 73: 135-148

van den Bout I, Divecha N (2009) PIP5K-driven Ptdlns(4,5)P2 synthesis: regulation and cellular functions. Journal of cell science 122: 3837-3850

van Horck FP, Lavazais E, Eickholt BJ, Moolenaar WH, Divecha N (2002) Essential role of type I(alpha) phosphatidylinositol 4-phosphate 5-kinase in neurite remodeling. Current biology : CB 12: 241-245

Wang Y, Lebowitz D, Sun C, Thang H, Grynpas MD, Glogauer M (2008a) Identifying the relative contributions of Rac1 and Rac2 to osteoclastogenesis. Journal of bone and mineral research : the official journal of the American Society for Bone and Mineral Research 23: 260-270

Wang Y, Lian L, Golden JA, Morrisey EE, Abrams CS (2007) PIP5KI gamma is required for cardiovascular and neuronal development. Proceedings of the National Academy of Sciences of the United States of America 104: 11748-11753 
Wang Y, Litvinov RI, Chen X, Bach TL, Lian L, Petrich BG, Monkley SJ, Kanaho Y, Critchley DR, Sasaki $T$ et al (2008b) Loss of PIP5KIgamma, unlike other PIP5KI isoforms, impairs the integrity of the membrane cytoskeleton in murine megakaryocytes. The Journal of clinical investigation 118: 812-819

Wang YJ, Li WH, Wang J, Xu K, Dong P, Luo X, Yin HL (2004) Critical role of PIP5KI\{gamma\}87 in InsP3-mediated $\mathrm{Ca}(2+)$ signaling. The Journal of cell biology 167: 1005-1010

Wang ZQ, Ovitt C, Grigoriadis AE, Mohle-Steinlein U, Ruther U, Wagner EF (1992) Bone and haematopoietic defects in mice lacking c-fos. Nature 360: 741-745

Zhu T, Chappel JC, Hsu FF, Turk J, Aurora R, Hyrc K, De Camilli P, Broekelmann TJ, Mecham RP, Teitelbaum SL et al (2013) Type I phosphotidylinosotol 4-phosphate 5-kinase gamma regulates osteoclasts in a bifunctional manner. The Journal of biological chemistry 288: 5268-5277

\section{Figure legends}

Figure 1 PIP5k1ß-deficient mice exhibit an osteoporotic bone phenotype. (A) Representative micro-CT images of femurs in WT or PIP5k1 $\beta$ knockout 2-month-old male mice. (B-F) Bone mineral density (BMD), Bone volume per tissue volume (BV/TV), trabecular number (Tb.N.), trabecular bone thickness (Tb.Th.) and trabecular separation (Tb.Sp.) were assessed from the micro-CT measurements $(n=10)$. $(\mathrm{G})$ The hematoxylin/eosin (H\&E) (up) and TRAP staining (down) of histological section of proximal tibiae. Bars, $100 \mu \mathrm{m} .(\mathrm{n}=10)$. (H-I) Analysis of trabecular bone area and quantification of N.Oc/BS (osteoclast number/ bone surface) in G. (J-K) Representative images of new bone formation $(\mathrm{J})$ and quantification of MAR $(\mathrm{K})$ as assayed by calcein double labeling. $(n=6)$. (L) Serum concentration of CTx-I in WT or PIP5k1 $\beta$ knockout mice $(n=6)$. $(M-N)$ The expression of CTSK in WT or PIP5k1 $\beta$ knockout mice shown by immunohistochemistry $(\mathrm{M})$ and the staining density were quantified by ImageJ $(\mathrm{N})$. ( $\mathrm{n}=6$ per group) (O) Serum concentration of OCN in WT or PIP5k1 $\beta$ knockout mice $(n=6)$ (Data represent as means \pm S.D. of three independent experiments. $* \mathrm{P}<0.05 ; * * \mathrm{P}<0.01 ; * * * \mathrm{P}<0.001$.)

Figure 2 PIP5k1 $1 \beta$ expression was increased during RANKL-induced osteoclast differentiation and PIP5k1ß-deficient osteoclasts exhibit normal 
morphology. (A) cDNA from preosteoclasts and mature osteoclasts were subjected to Micro array assay and the expression levels of PIP5k family genes and osteoclasts master genes were detected. The heat map is ordered by degree of differential expression of the indicated genes between preosteoclasts and mature osteoclasts. (B) RT-PCR detection for the expression levels of PIP5k1 $\beta$, TRAP, VATPased2 during osteoclastogenesis and 18s RNA was used as control. (C) Bone marrow cells from WT or PIP5k1 $\beta^{-/-}$mice were treated by $15 \mathrm{ng} / \mathrm{ml} \mathrm{M-CSF}$ for two days and the cells adhesion to the cell plates (named BMMs) were underwent osteoclastogenesis by stimulation with $20 \mathrm{ng} / \mathrm{ml} \mathrm{M-CSF}$ and $75 \mathrm{ng} / \mathrm{ml}$ RANKL for 7 days. The actin ring formation, Vinculin, and Rac1 expression and colocalization with F-actin were assessed by classical confocal microscopy. F-actin was stained with phalloidin (green) and nuclear was stained with Hoechst 3342. (All the data were confirmed by three independent experiments.)

\section{Figure 3 Deficiency of PIP5k1ß enhanced osteoclast differentiation and bone} resorption. Bone marrow cells from WT or PIP $5 \mathrm{k} 1 \beta-/-$ mice were treated by $15 \mathrm{ng} / \mathrm{ml}$ M-CSF for two days and the cells adhesion to the cell plates underwent osteoclastogenesis by stimulation with $20 \mathrm{ng} / \mathrm{ml} \mathrm{M-CSF}$ and $75 \mathrm{ng} / \mathrm{ml}$ RANKL for the indicated number of days, the osteoclasts formation were detected by TRAP staining (A) and the osteoclasts number were counted (C) (n=3). (B, D-J) WT or PIP5k1 $\beta-/-$ BMMs were cultured with M-CSF (20 ng/mL) and RANKL (75 ng/mL) for 0, 1, 3, 5 or 7 days. PIP5k1 $\beta$, PIP5k1 $\alpha$, PIP5k1 $\gamma$ and osteoclast-specific gene expression (ACP5, Ctsk, ATPasev0d2, NFATc1, DC-STAMP and OSCAR) were analyzed by RT-PCR (B) and real-time polymerase chain reaction (PCR) and the results were normalized to the expression of GAPDH (D-J). All experiments were performed at least three times. (K) WT (K left) or PIP5k1 $\beta-/-$ (K right) BMMs were cultured on bovine bone slices with $20 \mathrm{ng} / \mathrm{ml} \mathrm{M-CSF}$ and $75 \mathrm{ng} / \mathrm{ml}$ RANKL for 8 days and the pit formation was detected by SEM and the pit formation rate was measured by Image $J$ and presented graphically (L) (All the experiments were repeated three times. Data represent as means \pm SD of three independent tests. $* \mathrm{P}<0.05$; $* * \mathrm{P}<0.01$; $* * * \mathrm{P}<0.001$ versus $\mathrm{WT})$.

Figure 4 Overexpression of PIP5k1ß arrests osteoclast formation. (A) WT 
BMMs were transduced with LV5-NC or gradually increased concentration of LV5-PIP5k1 $\beta$, and the expression of PIP5K1 $\beta$ was detected by RT-PCR. (B-C) BMMs transduced with LV5-NC or 50 MOI of LV5-PIP5k1 $\beta$ were cultured with RANKL and M-CSF for 0, 5 or 9 days. The cells were stained for TRAP activity and osteoclasts number were counted. (D-E) BMMs from A were cultured with RANKL and M-CSF for 9 days. The cells were stained for TRAP activity and osteoclasts number were counted. (Scale bar, $200 \mu \mathrm{m}$, all the experiments were repeated three times. Data represent as means $\pm \mathrm{SD}$ of three independent tests. $* * *, \mathrm{P}<0.001$.)

Figure 5 Deficiency of PIP5k1ß enhances proliferation, migration and NFATc1 nuclear localization in preosteoclasts. (A) The wound healing assay. WT or PIP5k1 $\beta-/-$ BMMs were cultured in 48-well plates and serum- and cytokine-starved for $12 \mathrm{~h}$; scratches were made and serum-free culture medium with M-CSF or both M-CSF and RANKL was added. Cells migrating to the scratches were monitored and images were taken at 0 and $17 \mathrm{~h}$ after wounding. (B) The migration rate was calculated according to the equation: percentage wound healing $=(($ wound length at $0 \mathrm{~h})-($ wound length at $17 \mathrm{~h})) /($ wound length at $0 \mathrm{~h}) \times 100$ (C) CCK8 assay was performed to text the proliferation rate of WT or PIP5k1 $\beta-/-$ BMMs under triggering with M-CSF, RANKL, or both. (D-E) Nuclear translocation of NFATC1 in WT or PIP5k1 $\beta-/-$ osteoclasts stimulating with M-CSF and RANKL was detected by immunofluorescence (D) and western blotting (E). (F) RAW264.7 cells stably transfected with an NFATC1 luciferase reporter construct were seeded in 48-well plates and maintained in the cell culture medium for $24 \mathrm{~h}$. The cells were then transduced with vector or pEGFP-PIP5k1 $\beta$ for 24 $\mathrm{h}$, followed by incubation with RANKL (100 $\mathrm{ng} / \mathrm{mL})$ for $4 \mathrm{~h}$. NFATC1 luciferase activity was measured (down) and PIP5k1 $\beta$ mRNA levels were detected via RT-PCR (up). (All experiments were repeated three times. Data represent as means \pm SD of triplicate tests. $* \mathrm{P}<0.01$; **, $\mathrm{P}<0.001$; \#\# $\mathrm{P}<0.01$; ***, $\mathrm{P}<0.0001$ versus $\mathrm{WT}$ ).

Figure 6 Deficiency of PIP5k1ß leads to increases of M-CSF- and RANKL-mediated MAPK, c-Fos and AKT signaling cascades.

(A-D) WT or PIP5k1 $\beta^{-/-}$BMMs were cultured with M-CSF $(20 \mathrm{ng} / \mathrm{mL})$ and RANKL (75 ng/mL) for 0, 1, 3, 5days. Expression of TRAF6, c-Fos, Grb2, TRAP and 
Ctsk were analyzed by western blotting (A), the band densitometry were quantified and normalized to $\beta$-actin using ImageJ and graphically (B-D). Actin served as loading controls. (E-J) Serum- and cytokine-starved WT and PIP5KI $\beta^{-/-}$macrophages were exposed to RANKL (100 ng/ml), Phosphorylated AKT, JNK, ERK, p38 were immunoblotted with time. Actin served as loading controls, and the expression levels were analyzed by ImageJ. (K-N) Serum- and cytokine-starved WT and PIP5KI $\beta^{-/-}$ macrophages were exposed to M-CSF (30 ng/ml). Phosphorylated AKT, ERK, JNK were immunoblotted with time. Actin served as loading control, and the expression levels were analyzed by ImageJ. (All experiments were repeated three times. Data represent means $\pm \mathrm{SD} . * \mathrm{P}<0.05 ; * * \mathrm{P}<0.01 ; * *, \mathrm{P}<0.001$ versus $\mathrm{WT})$.

Figure 7 PIP5k1 $\beta$ accelerates osteoblast differentiation. (A-B) The expression of OSX in WT or PIP5k1 $\beta$ knockout mice shown by immunohistochemistry (A) and the staining density were quantified by ImageJ (B). (n=6 per group) (C) WT BMSCs were transfected with vectors or LV3-PIP5k1 $\beta$ (knockdown) or LV5-PIP5k1 $\beta$ (overexpression) to obtain BMSCs that constantly knockdown or overexpress PIP5k1 $\beta$, the transfection ratio of LV5-PIP5k1 $\beta$ were shown. (D-K) BMSCs from $\mathrm{C}$ were cultured with osteogenic medium for the indicated times. RT-PCR was used to test PIP5k1 $\beta$, BSP, OSX, OCN expression for an indicated number of times (D-E) and quantitative real-time PCR was performed for the mRNA expression of PIP5k1 $\beta$, Alpl (ALP), Ibsp (BSP), SP7, OCN and Colla1 (F-K). (Data represent as means \pm SD of triplicate samples. cn, control cells treated with control medium; o and o-cn, cells overexpress PIP5k1 $\beta$ treated with control medium; cn-ob, control cells treated with osteogenic medium; o-ob, cells overexpress PIP5k1ßtreated with osteogenic medium.) (L) Cells from $\mathrm{C}$ cultured for 7 days with osteogenic medium were fixed and stained for ALP. (M) Cells cultured with osteogenic medium for 14 days were fixed and stained for Alizarin red and were quantified by densitometry at $548 \mathrm{~nm}$. (N) Western blot for osteoblasts marker genes for 7 and 14 days treatment with osteogenic medium and $\beta$-actin were used as loading control. (All experiments were performed triplicate. Data represent means $\pm \mathrm{SD}$ of triplicate samples. $* \mathrm{P}<0.05 ; * * \mathrm{P}<0.01 ; * * * \mathrm{P}<0.001$ versus control, cn, cells treated with control medium; om, cells treated with osteogenic 
medium.)

Figure 8 PIP5k1 $1 \beta$ accelerates osteoblast differentiation through activating p-Smad1/5/8 signaling. WT BMSCs were transfected with vectors or LV3-PIP5k1 $\beta$ (knockdown) or LV5-PIP5k1 $\beta$ (overexpression) to obtain BMSCs that constantly knockdown or overexpress PIP5k1 $\beta$. Then were underwent with control or osteogenic medium incubation for 7 or 14 days. (A) Western blot for p-smad1/5/8 after 7 and 14 days treatment with osteogenic medium and $\beta$-actin were used as loading control. (B) The band densitometry from A was analyzed with ImageJ and was shown graphically. (C) ALP and Alizarin Red S staining for BMSCs incubated with control or osteogenic medium with or without smad1/5/8 specific inhibitor LDN193189 for 7 and 14 days respectively. (D) The ALP staining density was analyzed using the software Image-Pro Plus and shown as graph. (E) The calcium precipitates in figure B were dissolved in 0.1 $\mathrm{N}$ sodium hydroxide and quantified by absorbance at $548 \mathrm{~nm}$ with a Tecan Safire2 microplate reader (Tecan, Durham, NC, USA) (F) Westernblotting for BMSCs incubated with control or osteogenic medium with or without smad1/5/8 specific inhibitor LDN193189 for 7 and 14 days respectively. Expression of p-smad1/5/8 and osteogenic marker genes were detected and analyzed with ImageJ. (All experiments were performed triplicate. Data represent means $\pm \mathrm{SD}$ of triplicate samples. $* \mathrm{P}<0.05$; ** $\mathrm{P}<0.01 ; * * * \mathrm{P}<0.001$ versus control, cn, cells treated with control medium; om, cells treated with osteogenic medium.)

Supplementary Figure 1. (A) The whole body of WT and PIP5k1 $\beta$ deletion mice. (B) Total RNA was isolated from bone, spleen, brain, thymus, lung, kidney, liver, epididymis, testis, heart, and muscle of WT mice. Quantitative real-time PCR was performed for the mRNA expression of PIP5k1 $\alpha, \beta$ and $\gamma$. (C-D) WT or PIP5k1 $\beta-/-$ BMMs were cultured with M-CSF (20 ng/mL) and RANKL (75 ng/mL) for 0, 1, 3, 5 or 7 days. $\beta 3$ Integrin expression was analyzed by real-time polymerase chain reaction (PCR) and the results were normalized to the expression of GAPDH (C) and western blotting (D). All experiments were performed at least three times $(* \mathrm{P}<0.05 ; * * \mathrm{P}<0.01)$ Supplementary Figure 2. (A) MTT assay to detect the proliferation rate of BMSCs constantly overexpress or knockdown of PIP5k1 $\beta$ and the control BMSCs. (B-C) 
Densitometry analysis of Fig.7N and the results were shown graphically. (All experiments were performed triplicate. Data represent means \pm SD of triplicate samples. $* \mathrm{P}<0.05 ; * * \mathrm{P}<0.01 ; * * * \mathrm{P}<0.001$ versus control, cn, cells treated with control medium; om, cells treated with osteogenic medium.) (D) Model of PIP5k1 $\beta$ to modulate bone homeostasis. On the one hand, RANKL binds to RANK then RANK recruit TRAF6, which leads to phosphorylation of JNK, Akt MAPK/Erk1/2 and MAPK/p38 signaling and activation of c-Fos, which are crucial for NFATC1 induction and activation. NFATC1 in turn initiates its own expression and activation. NFATC1 promotes osteoclastogenesis by promoting expression of osteoclastogenic genes such as DC-STAMP, Cathepsin k, OSCAR, ACP5, Atpasev0d2. PIP5k1 $\beta$ inhibits RANKL-induced MAPK, AKT activation and TRAF6, c-Fos expression; PIP5k1 $\beta$ also inhibits Grb2 expression during osteoclastogenesis. Grb2 promotes osteoclasts proliferation and formation. In this way, PIP $5 \mathrm{k} 1 \beta$ inhibits osteoclastogenesis to modulate bone resorption. On the other hand, PIP5k1 $\beta$ promotes expression of osteogenic genes such as ALP, OSX, RUNX2, OCN, BSP, col1 $\alpha 1$ to enhance bone formation through activating smad1/5/8 signaling. 
B M-CSF 99090996 Days $\begin{array}{llllll}\text { RANKL } & 0 & 1 & 3 & 5 & 7\end{array}$

A

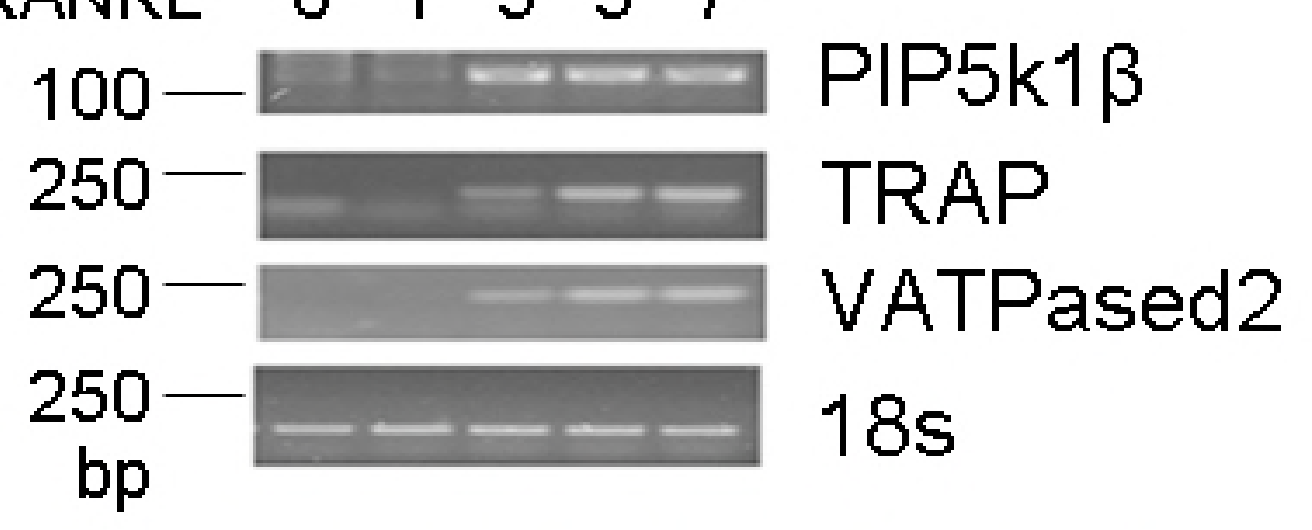

Pip5k1 $\alpha$

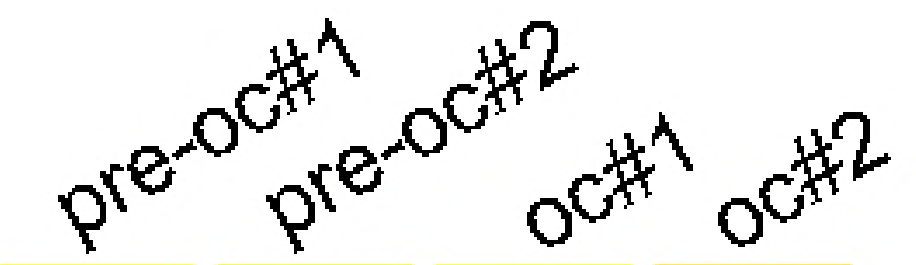

Pip5k1 $\beta$ Pip5k1y Pip5k111

Pip5k3

Acp5
DC-stamp Oscar Src Calcr Ctsk Mmp9

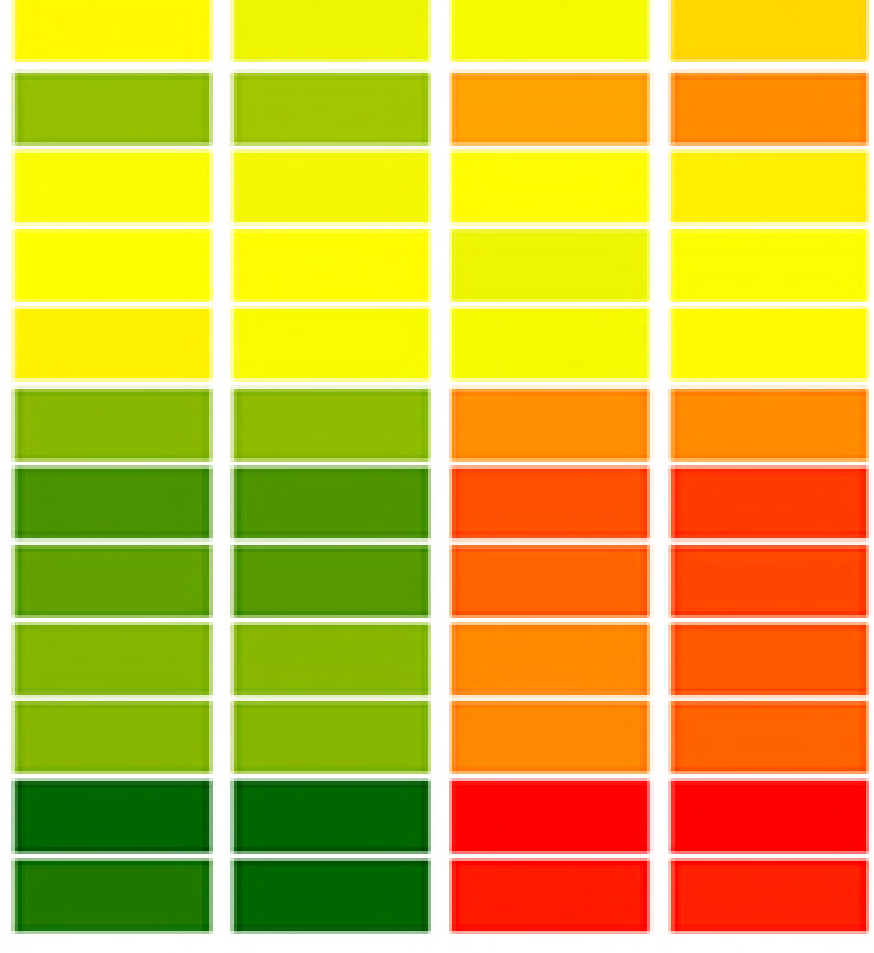

C

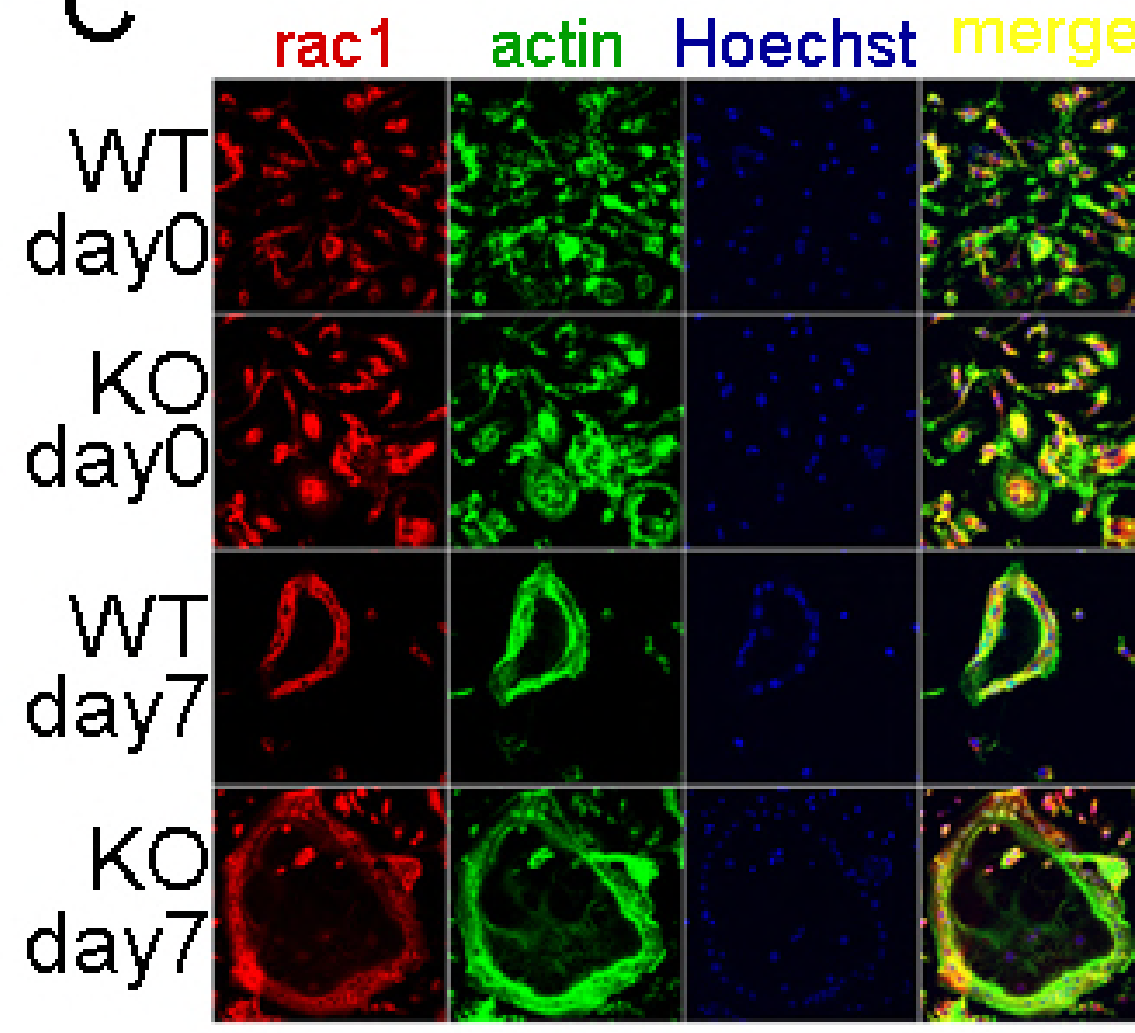

vinculin actin Hoechst merge

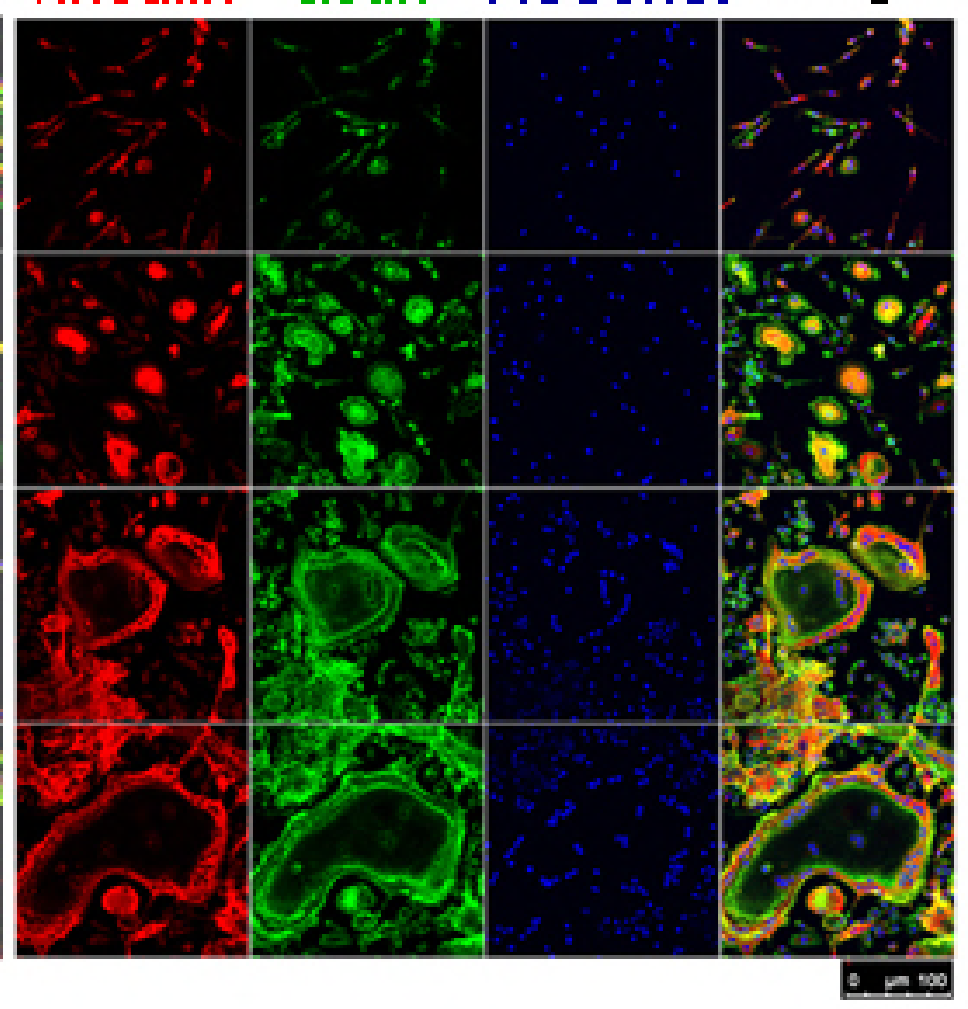




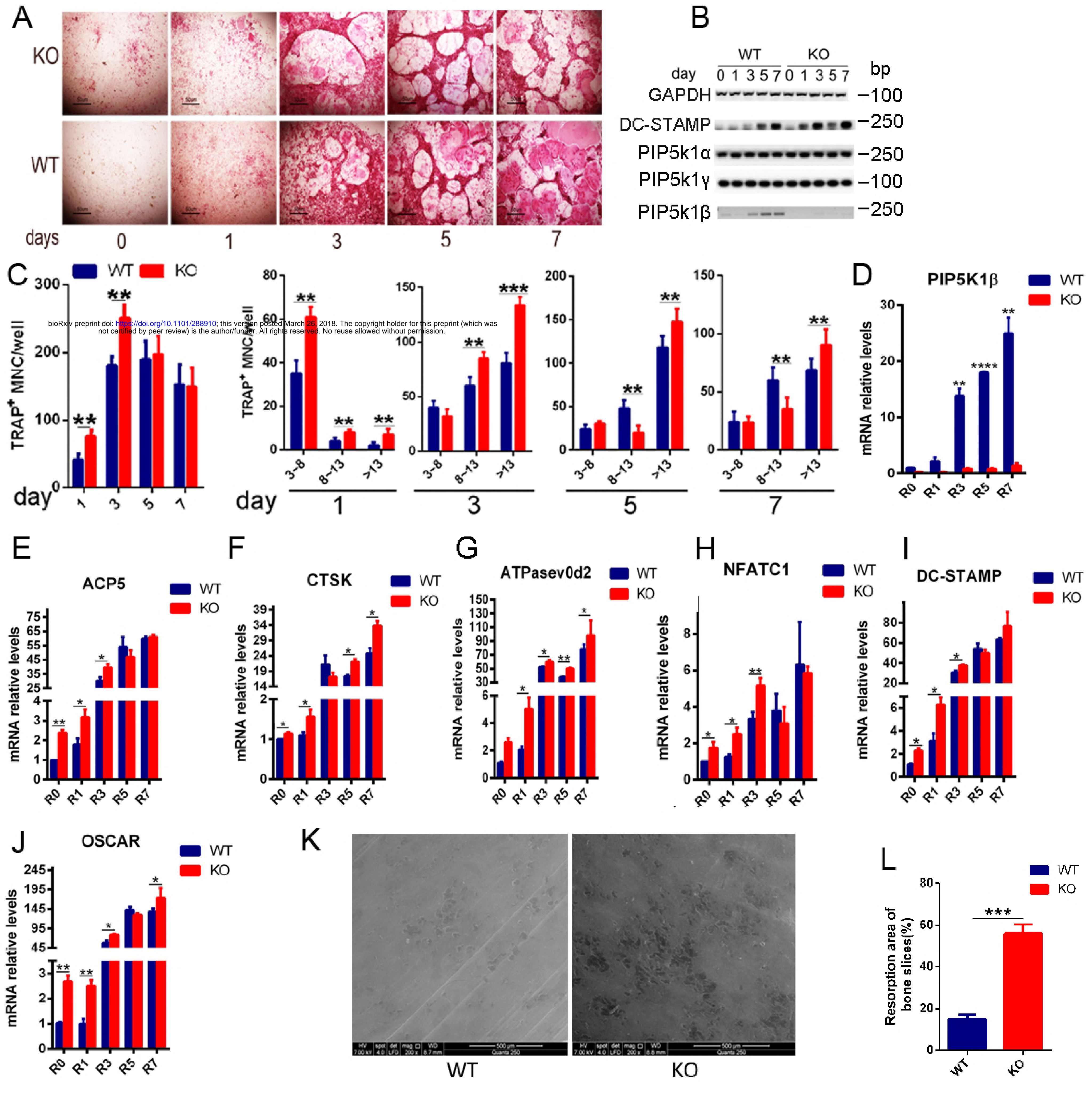


A

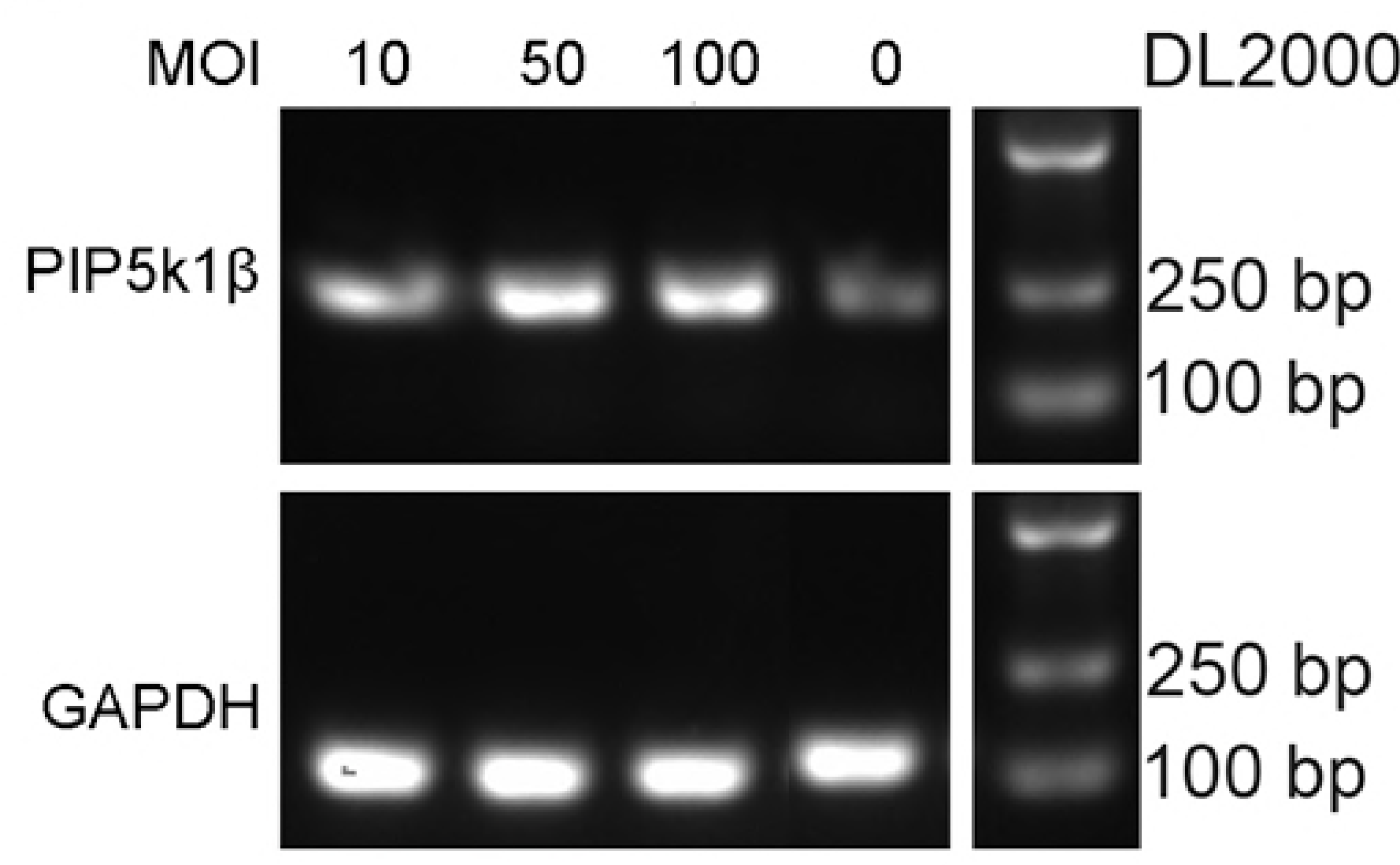

B

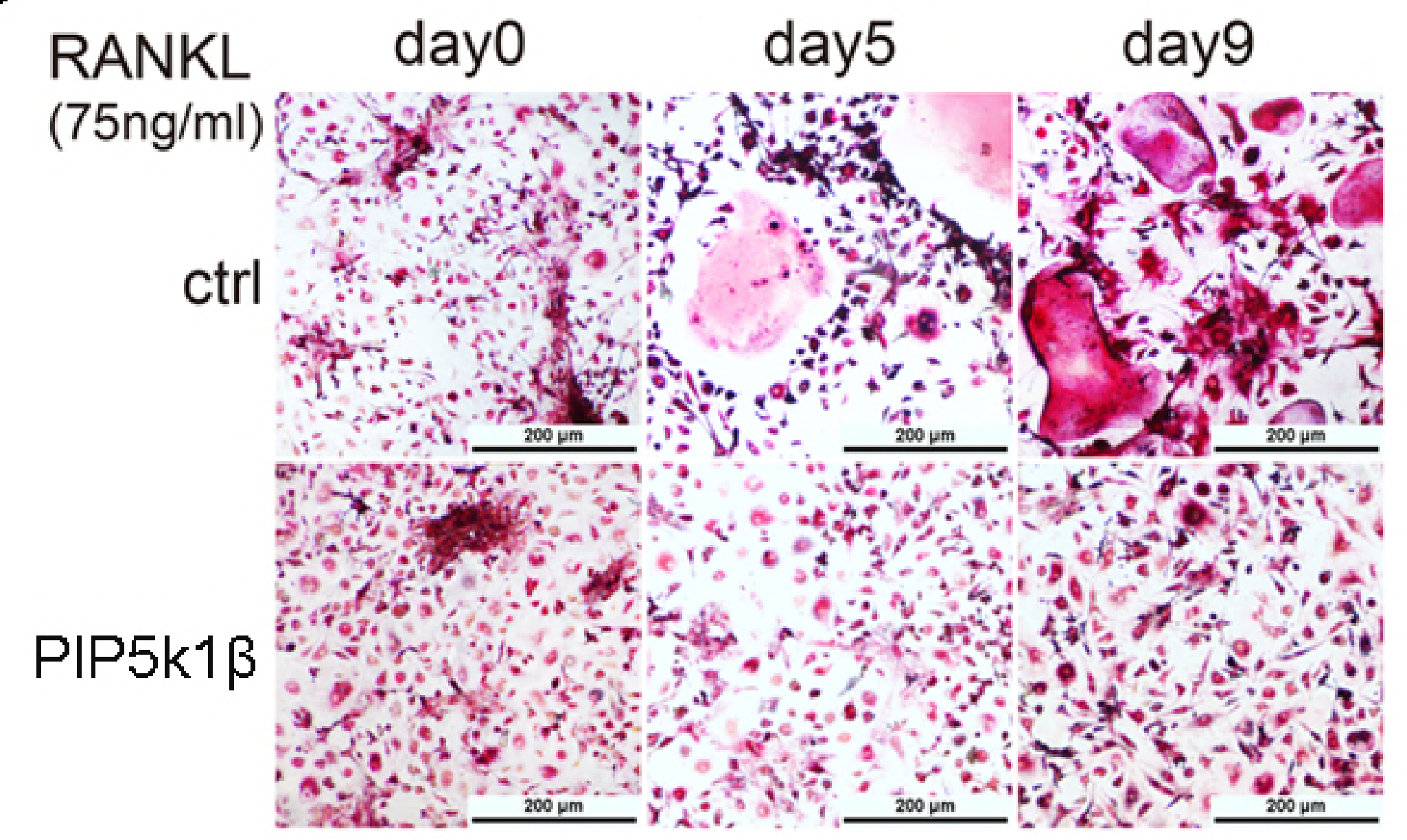

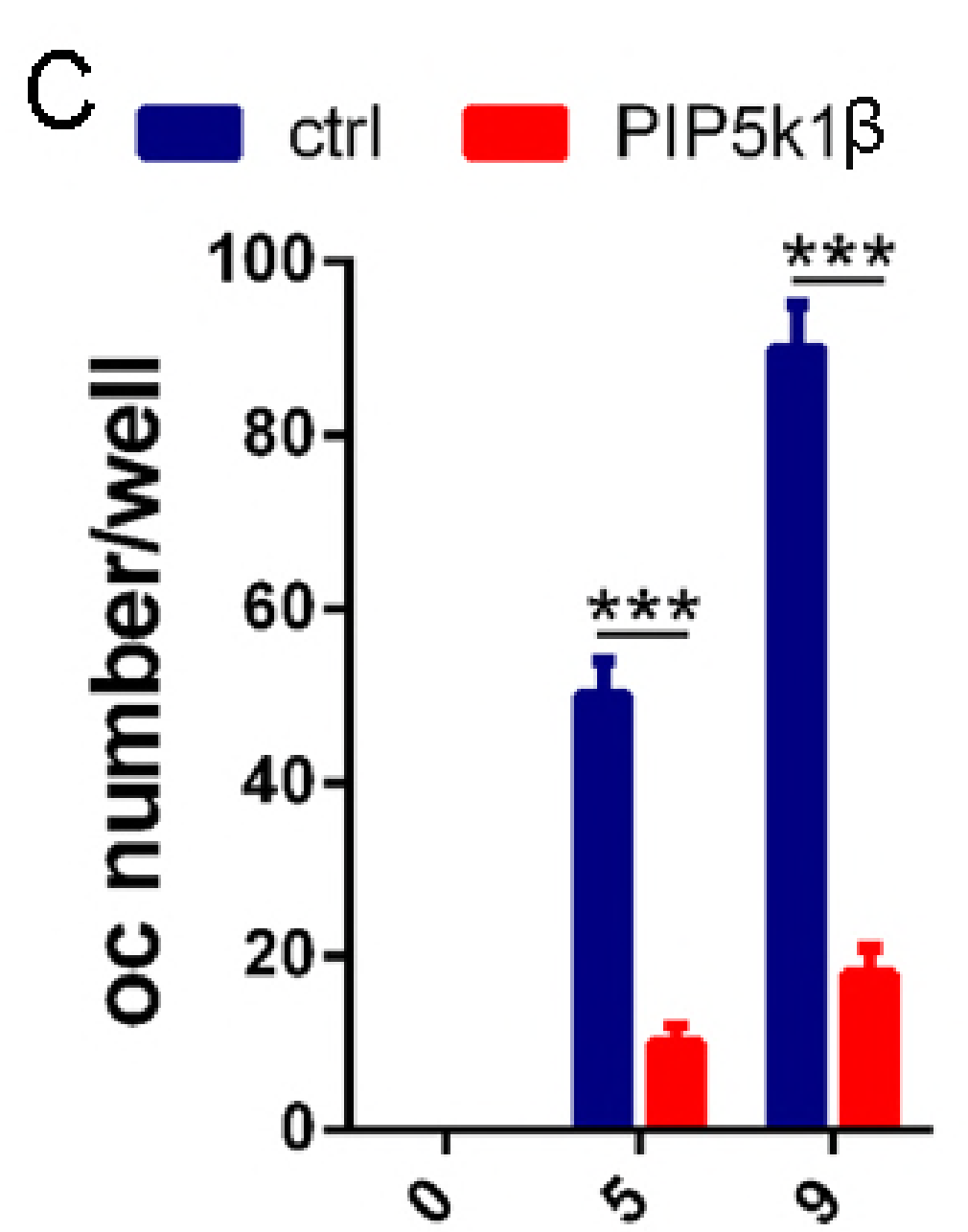

D $\mathrm{MOI}$

10

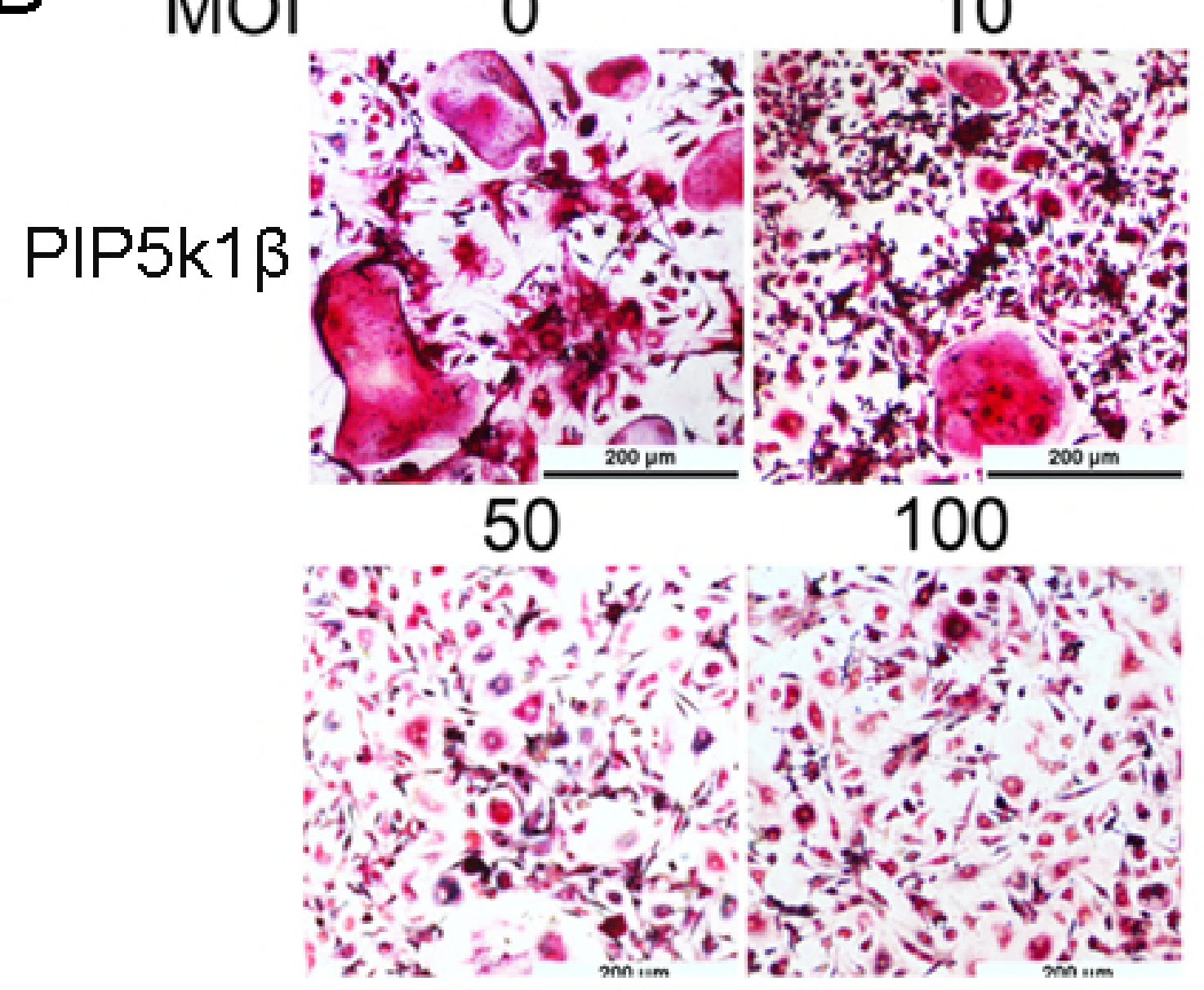

$E$

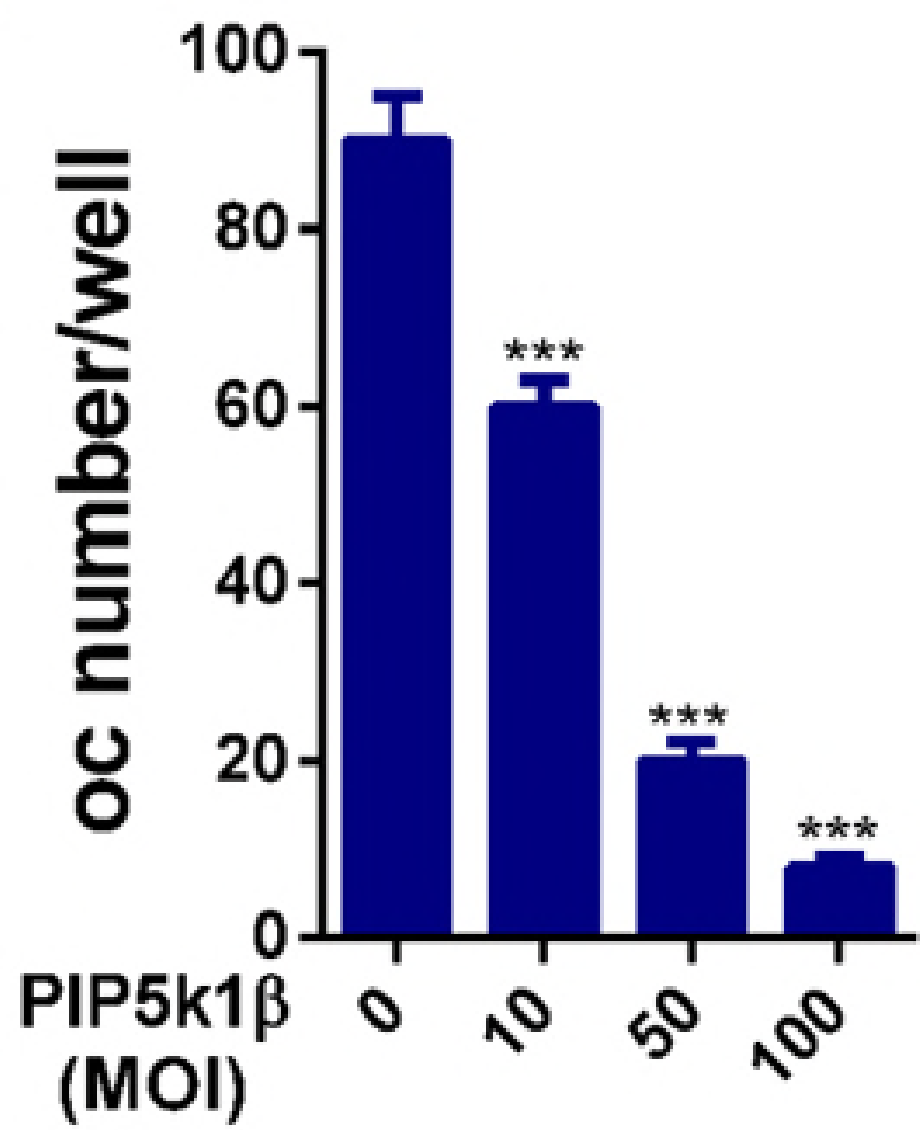


A

$\beta$-actin - - - - - $-43 \mathrm{kDa}$ GRB2 $-25 \mathrm{kDa}$ c-Fos - - $-60 \mathrm{kDa}$ TRAF6 - - - - - $-60 \mathrm{kDa}$

TRAP -36 kDa ctsk $-\infty-43 \mathrm{kDa}$

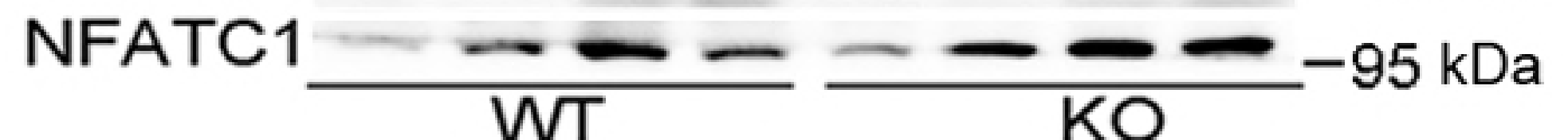
$\begin{array}{lllllllll}\text { RANKL(d) } & 0 & 1 & 3 & 5 & 0 & 1 & 3 & 5\end{array}$

$E$

p-AKt - - D - - -

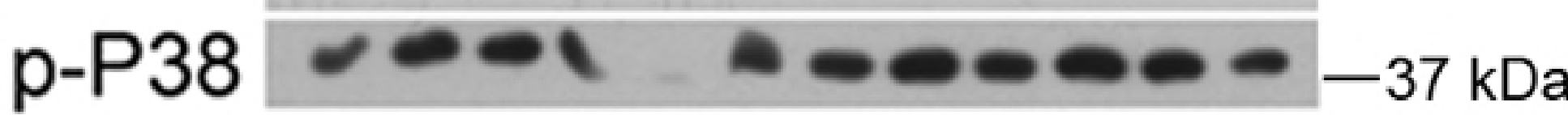

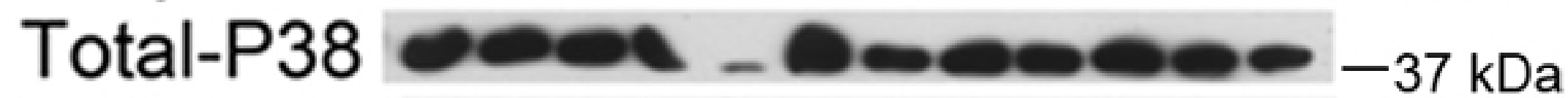
actin - - -

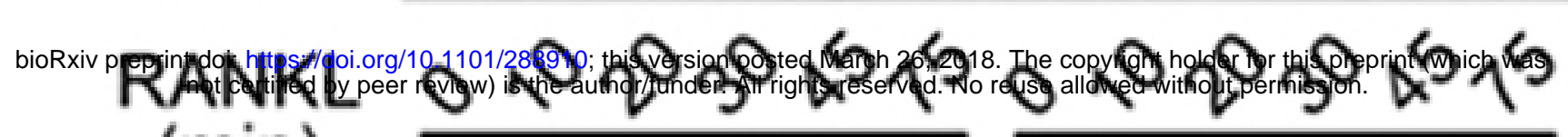

(min) $\mathrm{WT}$

G

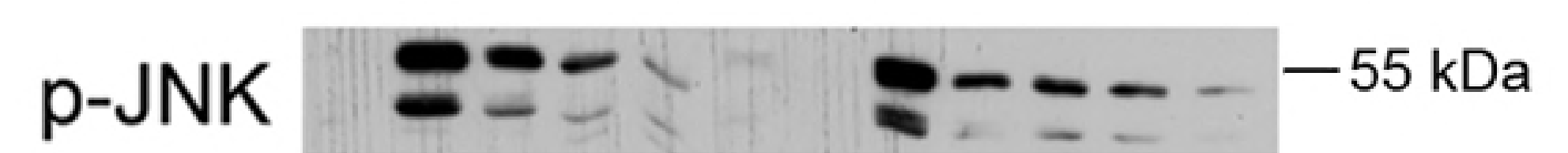

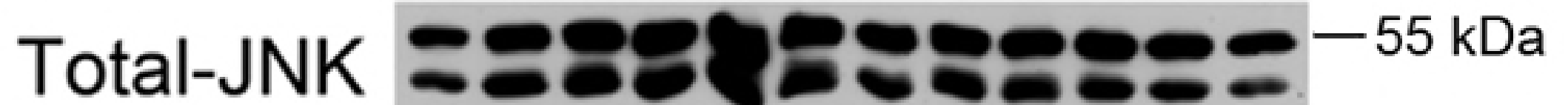

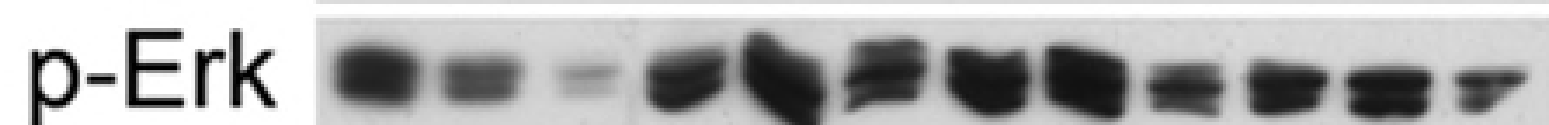

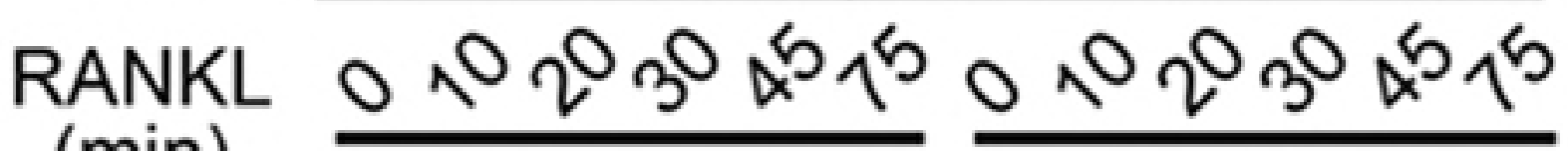

(min) $\frac{W T}{K O}$

K

p-AKt 은 으-- $-55 \mathrm{kDa}$ p-JNK 으=ㅡ-

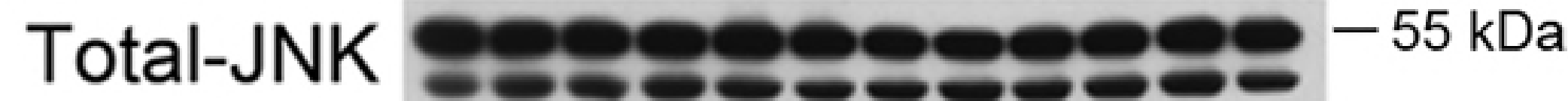
p-Erk $=\mathbf{z}=\mathbf{z}=\mathbf{=}==-37 \mathrm{kDa}$

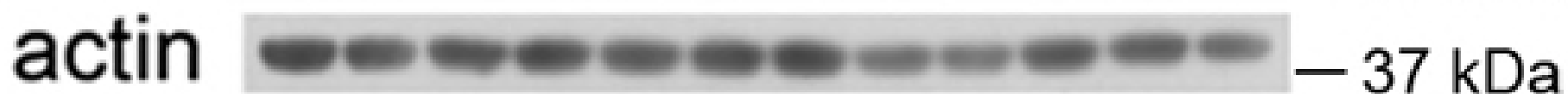

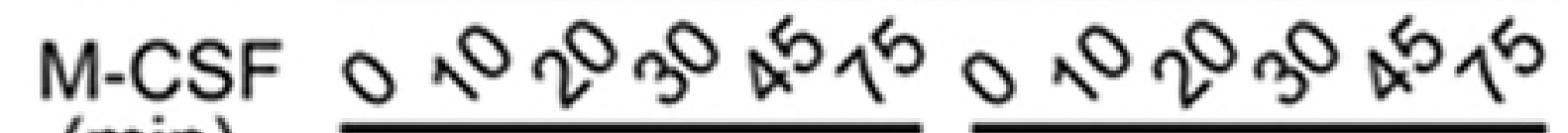

(min)

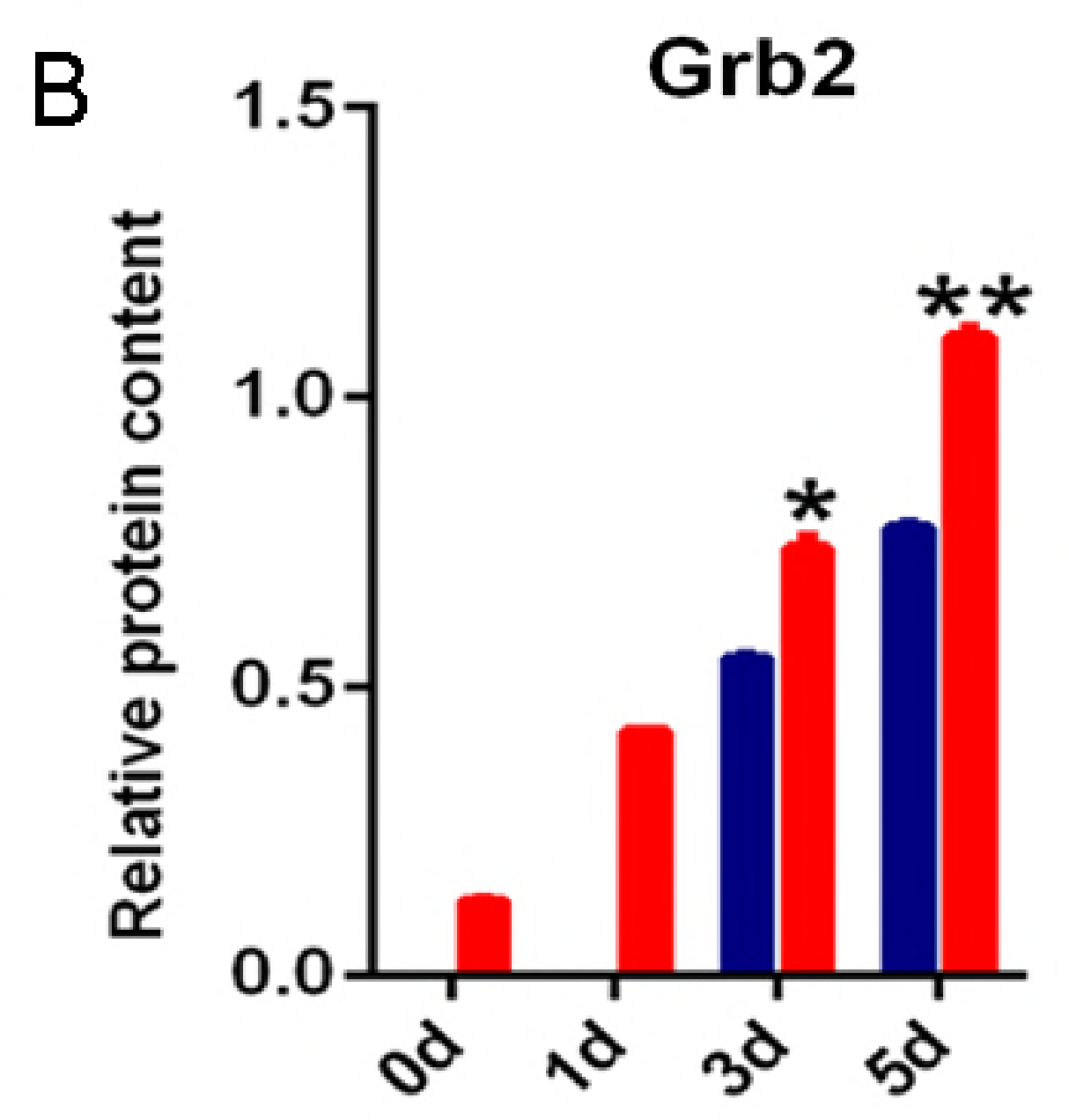

c-fos

TRAF6

NFATC1
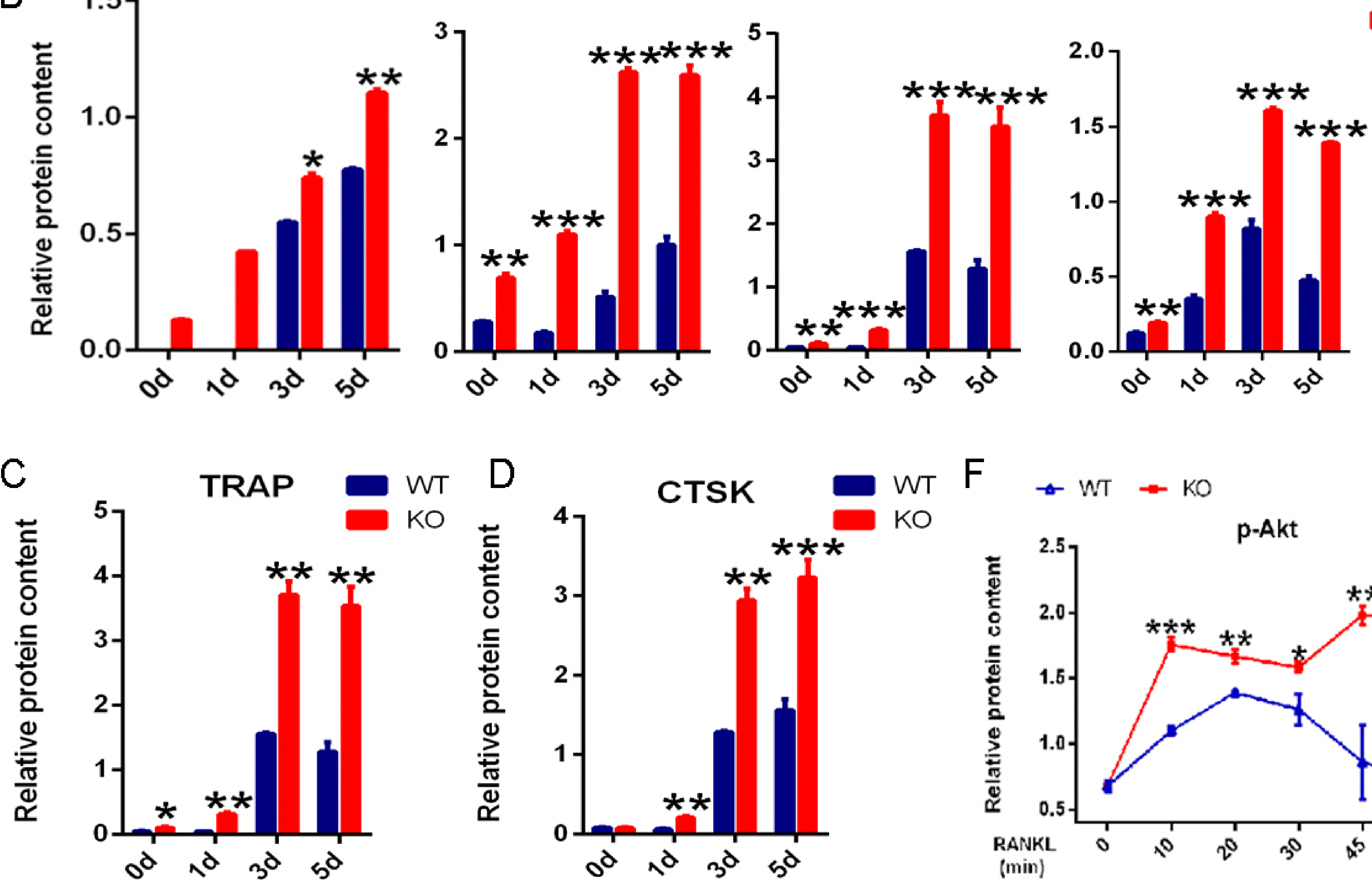

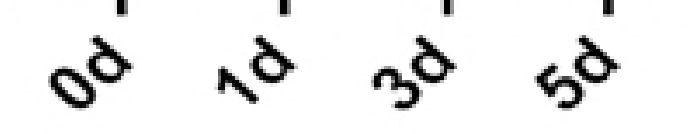
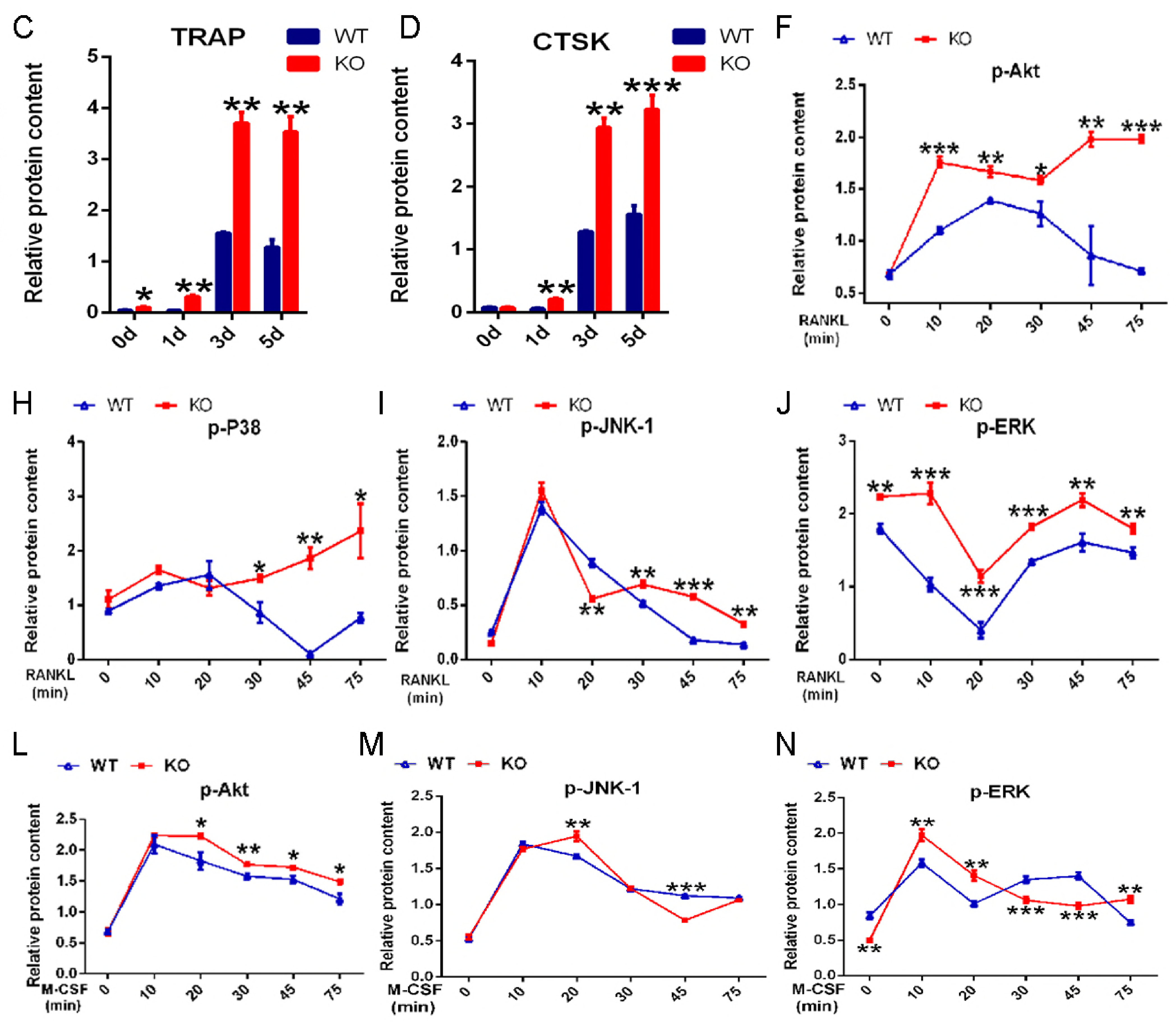

$\mathrm{N}$

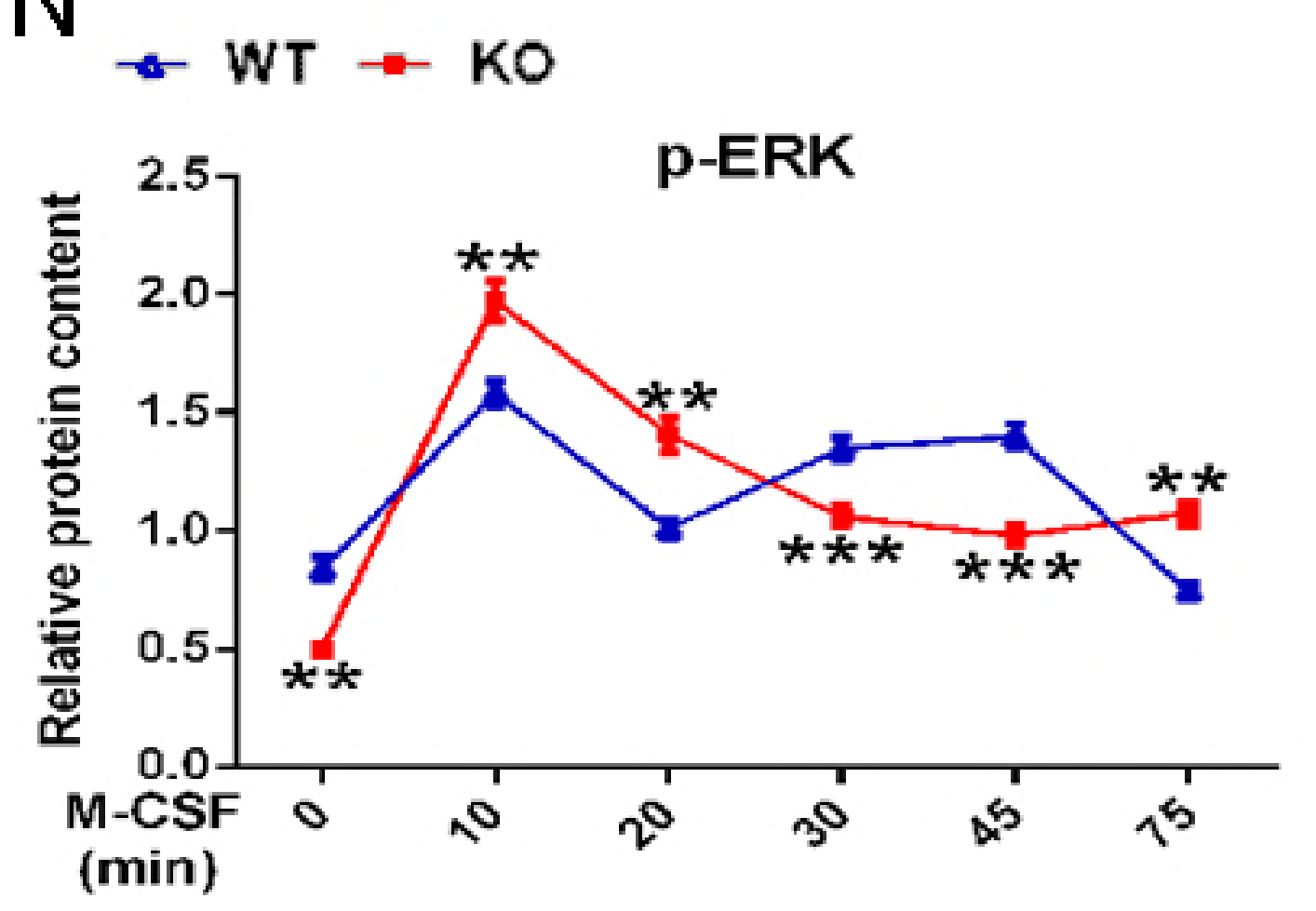


A

A $\quad W T$

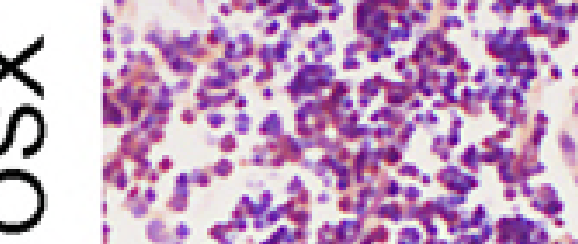

\% W

nive 0 .

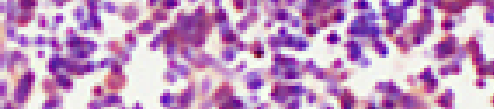

L 3 ,

C

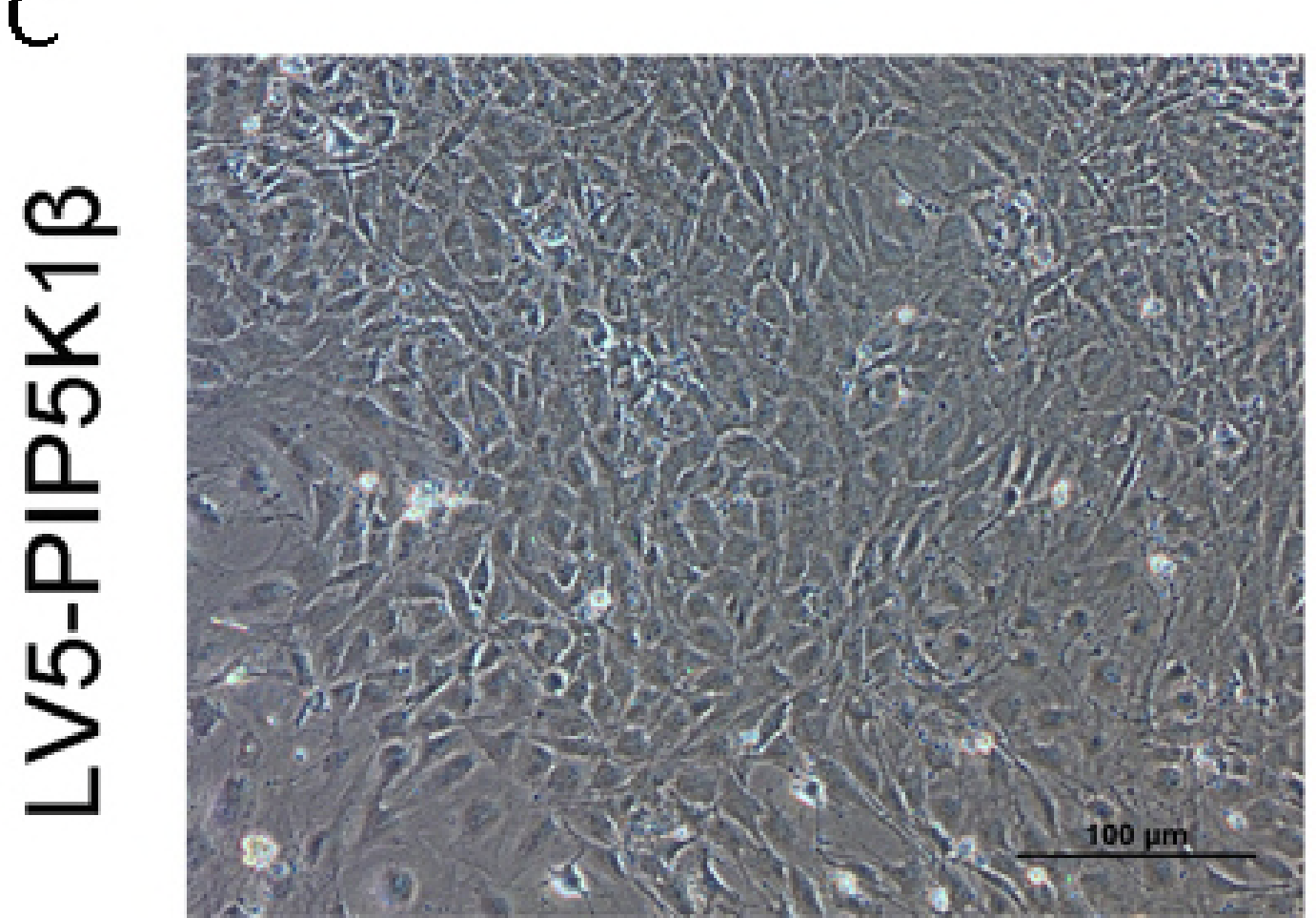

$\mathrm{KO}$
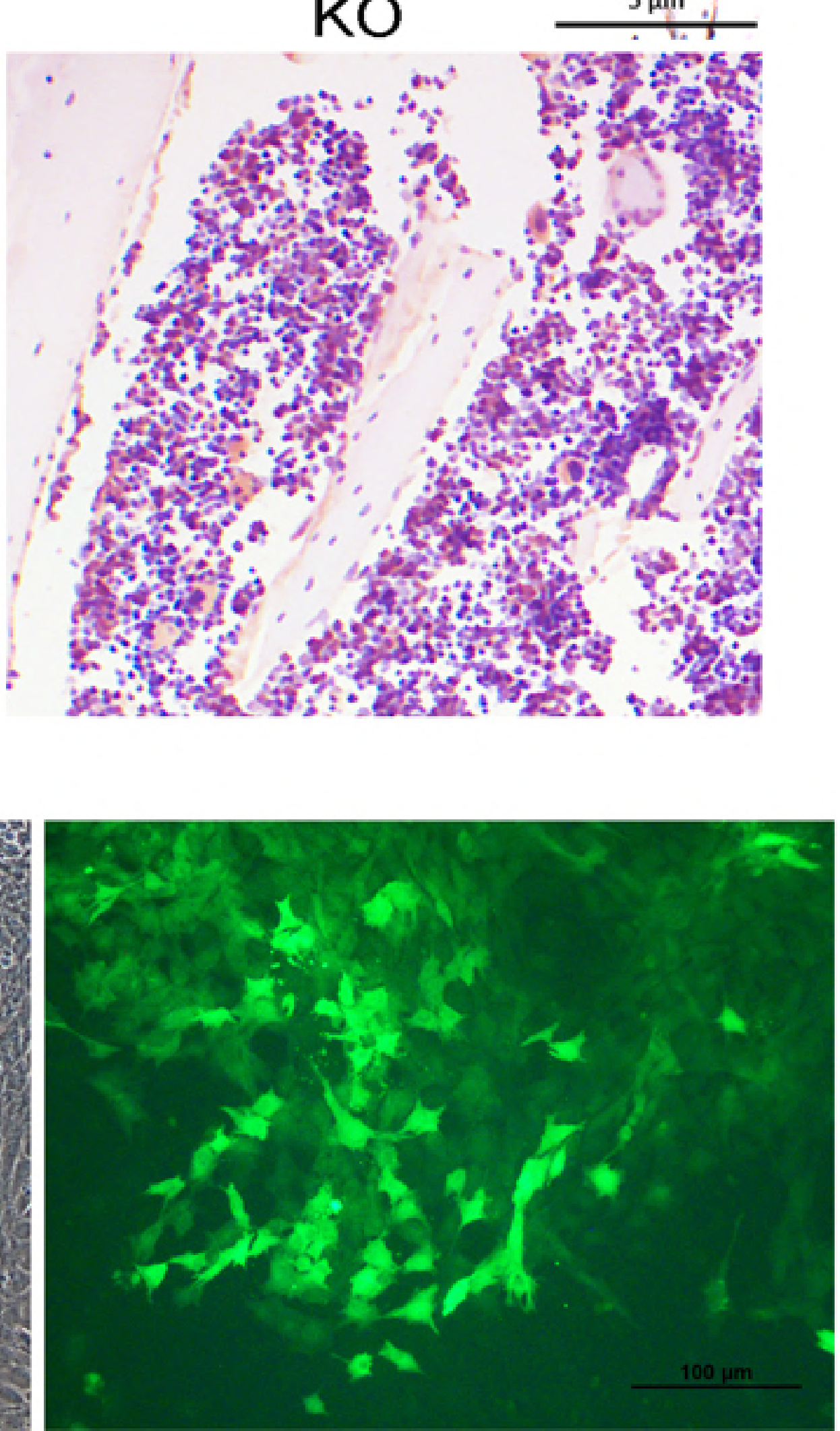

B

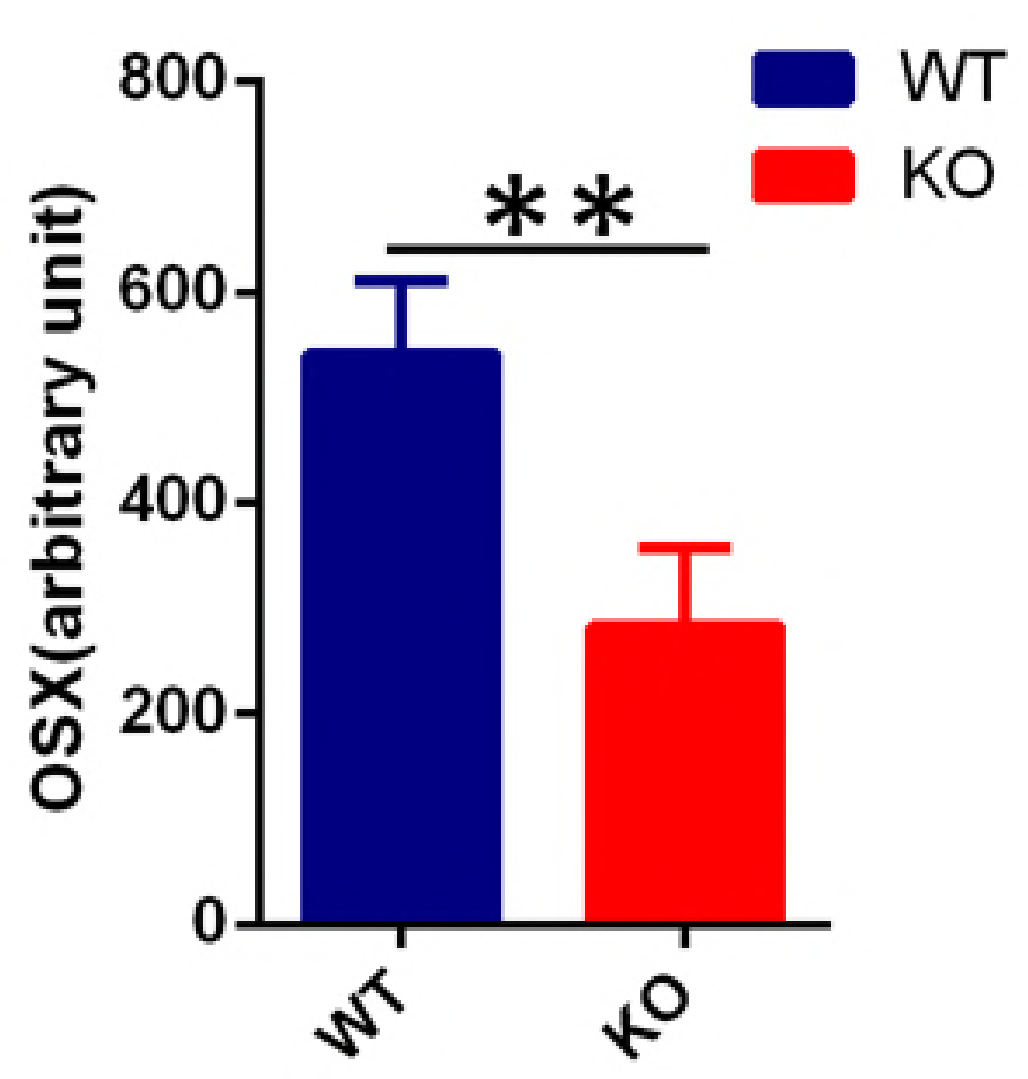

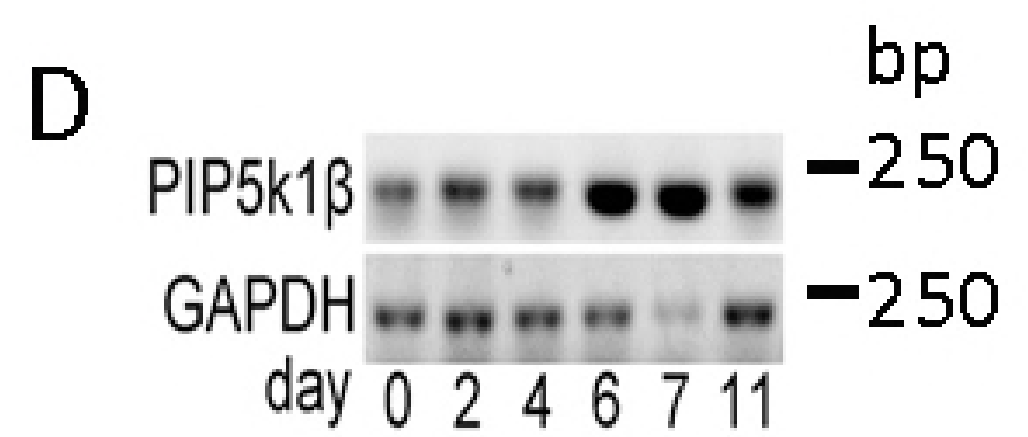

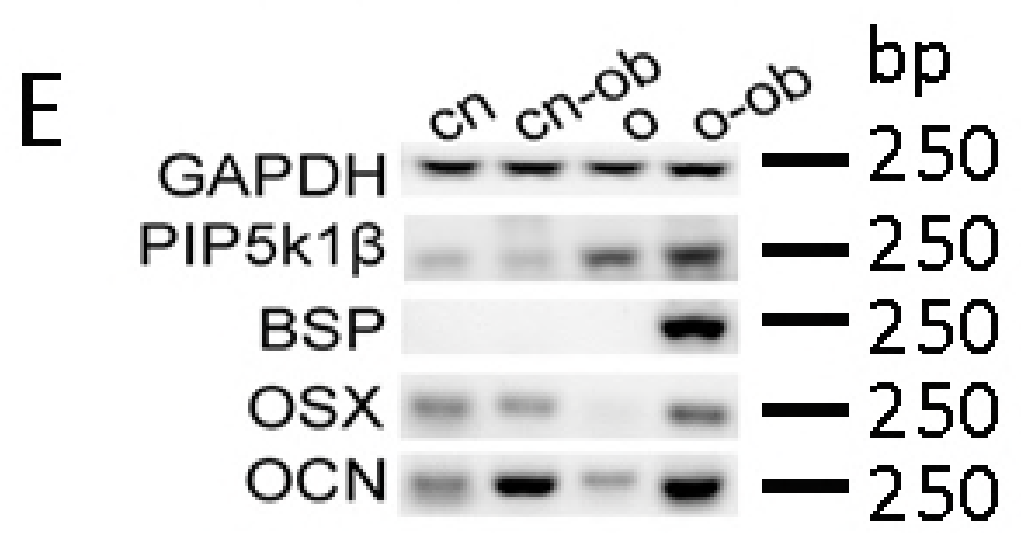
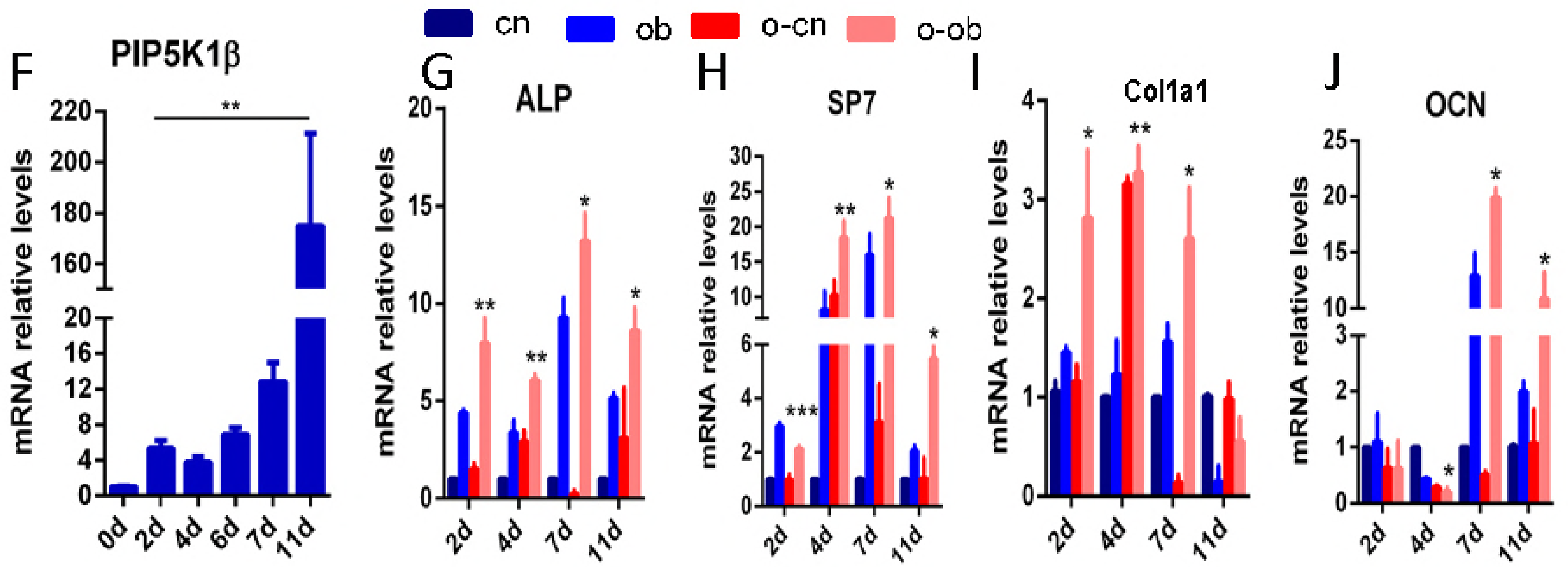

K
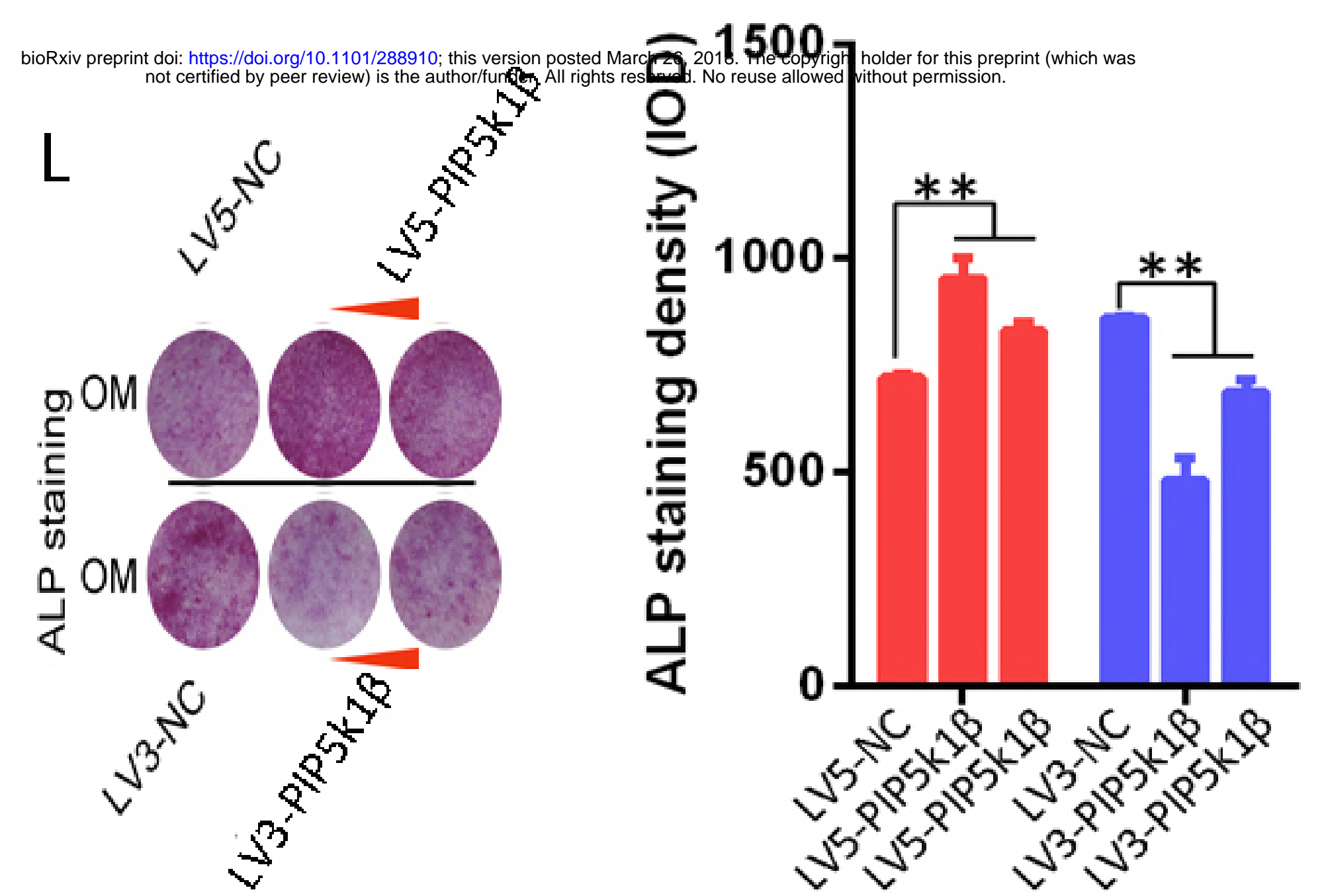

$N$
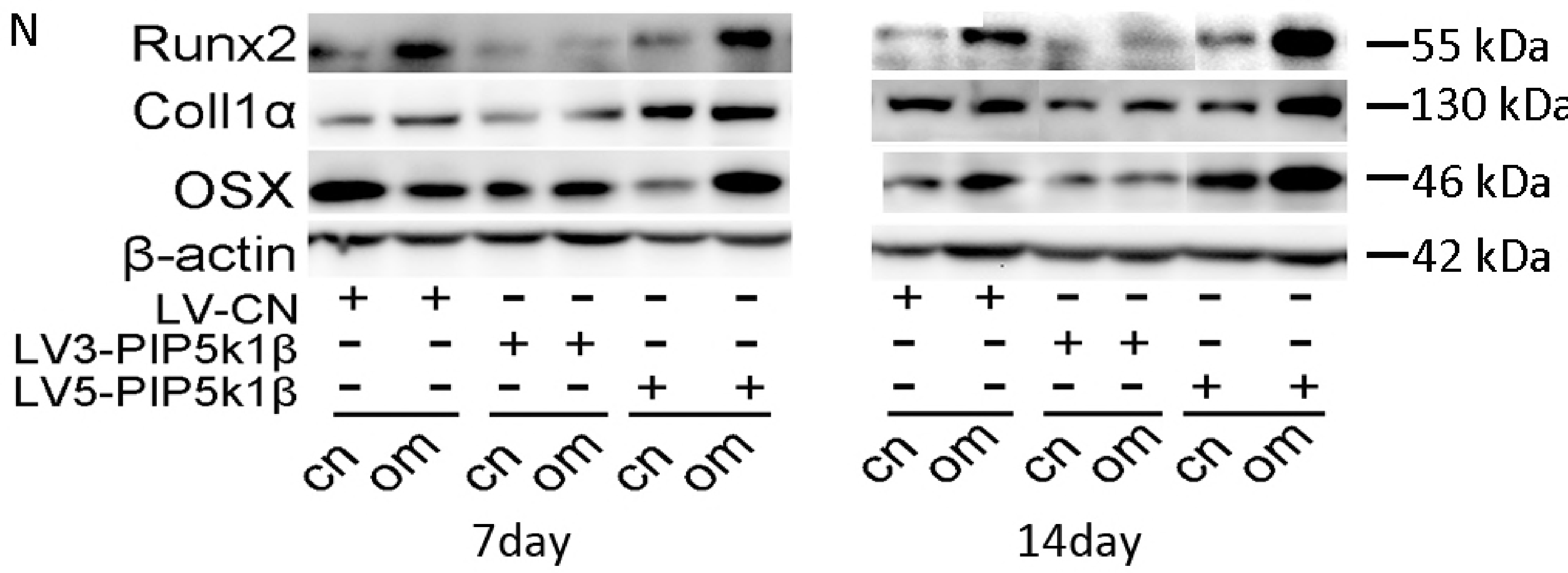
A

$\mathrm{p}-\mathrm{Smad} 1 / 5 / 8$

$\beta$-actin

$\mathrm{LV}-\mathrm{CN}$

LV3-PIP5k1 $1 \beta$

$++-$

LV5-PIP 5k1 $1 \beta$

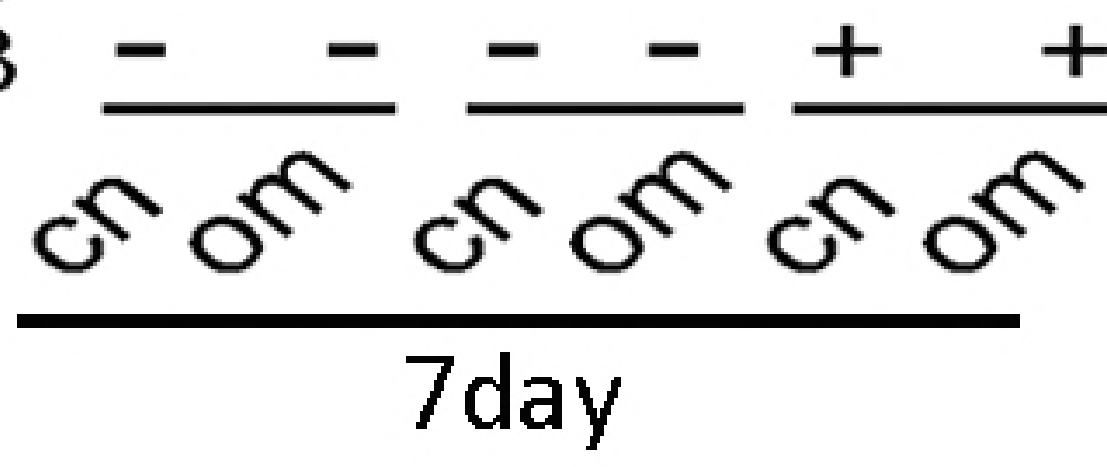

B

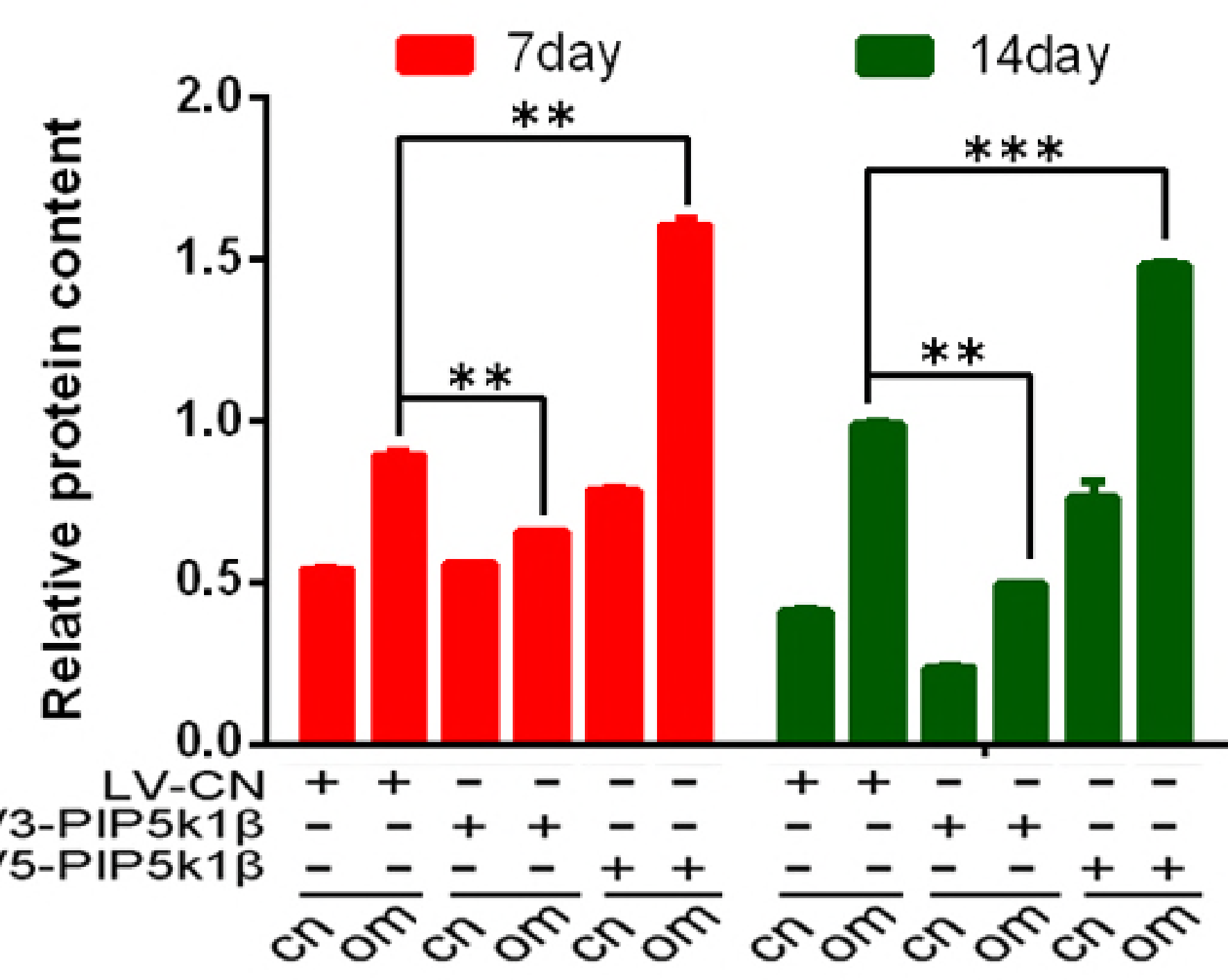

C

ALP staining

Alizarin staining

$\operatorname{LDN}(0.1 \mathrm{uM})$

$\mathrm{F}$

$\mathrm{p}-\mathrm{Smad} 1 / 5 / 8$

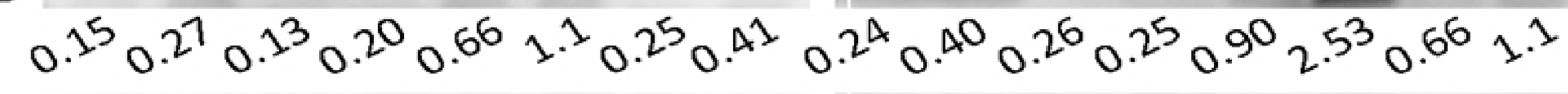

COL1a1- - - - - - - - - - - - - - - $0.4^{4} 0.6^{8} 0.3^{5} 0.5^{4} 0.8^{4} 1.00 .3^{3} 0 . .^{6} 0.5^{8} 0 . .^{55} 0.2^{9} 0.6^{60} 0.902 .10 .8^{4} 1^{4}$

Runx2 =- - - - - - - - - - - -

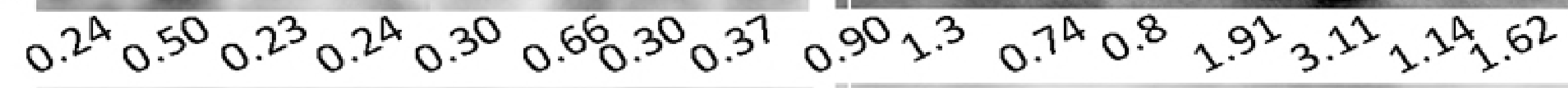

OSX - - - - - - - - - - -

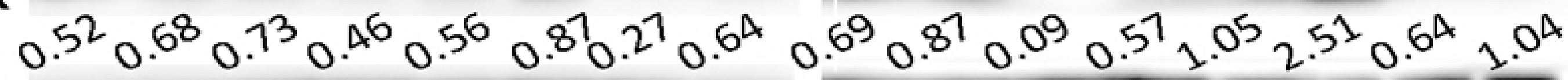

$\beta$-actin $\longrightarrow+22 \mathrm{kDa}$

$\operatorname{LDN}(0.1 \mathrm{uM})$

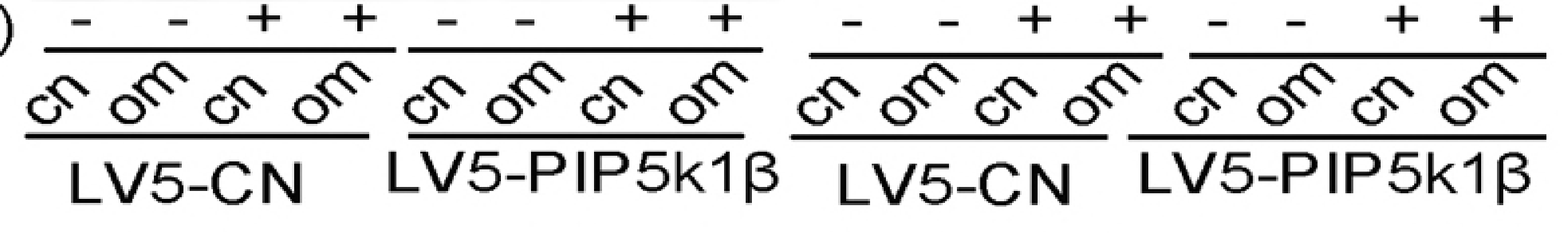

